\title{
Short-hairpin RNA-guided single gene knockdown reverses triple-negative breast cancer
}

Dong-Mei Chen ${ }^{1}$, Zhu-Qing Shi ${ }^{2}$, Li-Li Tan ${ }^{1}$, Yan-Ping Chen ${ }^{1}$, Chang-Qing Li ${ }^{1}$, Qi Wang ${ }^{3}$, $\mathrm{He} \mathrm{Li}^{4}$, Meng-Le Zhang ${ }^{4}$, Jian-Ping Song ${ }^{1 *}$, Qin $\mathrm{Xu}^{1 *}$, Qing-Ping Zeng ${ }^{1 *}$

1 Institute of Tropical Medicine, Guangzhou University of Chinese Medicine, Guangzhou, China

2 Science and Technology Industrial Park, Guangzhou University of Chinese Medicine, Guangzhou, China

3 Institute of Clinical Pharmacology, Guangzhou University of Chinese Medicine, Guangzhou, China

4 School of Basic Course, Guangdong Pharmaceutical University, Guangzhou, China

* Corresponding authors

\section{Abstract}

\section{Background}

The origin of breast cancer remains poorly understood. Here, we testify a putative mechanism of "breast cancer origin from inducible nitric oxide synthase (iNOS) activation and oestrogen receptor alpha $(\mathrm{ER} \alpha)$ inactivation", which are classified as the essential outcomes of chronic inflammatory responses.

\section{Methods}

To reverse breast cancer status, iNOS was downregulated by short-hairpin RNA (shRNA)-guided NOS2 knockdown from human triple- negative breast cancer (TNBC) cells. To re-enact breast cancer origin, ER $\alpha$ was downregulated by shRNA-directed ESR 1 knockdown in human mammary epithelial cells.

\section{Results}

Upon NOS2 knockdown from HCC1937 cells, the specific TNBC transcription factor genes, RUNX1 and BCL11A, were downregulated, hypoxia was compromised, Warburg effects were attenuated, and tumourigenic proliferation was halted, accompanied by an increase in the tumour marker cyclin D1 (CD1) and a decrease in the tumour suppressor cyclin-dependent kinase inhibitor (CKI). In contrast, ESR 1 knockdown from MCF-10A cells led to upregulated $B C L 11 A$ and $R U N X 1$ expression, augmented hypoxic responses, pronounced Warburg effects, and enhanced PI3K/Akt/mTOR signalling, together with low levels of CD1 and high levels of CKI 
induction.

\section{Conclusions}

Breast cancer should originate from inflammatory signalling, during which iNOS activation and ER $\alpha$ inactivation elicit hypoxia, oxidation, mitochondrial dysfunction, and breast cancer-like hyperplasia, demonstrating that iNOS inhibitors and ER $\alpha$ activators represent promising candidate prodrugs enabling breast cancer prevention in the early stage.

\section{Keywords}

breast cancer - gene knockdown - pro-inflammation - nitric oxide tumour origin

\section{Background}

Breast cancer affects approximately $12 \%$ of women worldwide and affected 2.8 million American women in 2015 [1]. Hormone receptor-positive $\left(\mathrm{HR}^{+}\right)$ breast cancer constitutes more than $70 \%$ of patients among postmenopausal women $[2,3]$. The incidence of $\mathrm{HR}^{+}$breast cancer has been anticipated to increase by $4 \%$ annually between 2011 and 2030 in women aged $70-84$ years old and $1.6 \%$ among women aged $50-69$ years old [4]. While $2-3 \%$ of the family-transmitted and early-onset patients can be attributed to the germ-line mutation genes $B R C A 1$ and $B R C A 2$ [5], most sporadic and late-onset clinical cases originate from versatile risk factors.

Although the aetiological initiators of breast cancer remain to be identified, the female hormones oestrogens, mainly including estrone (E1), estradiol (E2) and estriol (E3), have been postulated to promote mammary tumourigenesis via activation of oestrogen receptor (ER)-induced downstream pro-mitogenic transcriptional mechanics [6]. The ER-independent effects of oestrogens have been shown to also influence breast tumour development in concert with ER-dependent effects [7]. Recently, evidence from clinical and experimental data has emerged to link the gut microbiota to breast cancer [8]. This relevance was built on 40 years of literature regarding excreted oestrogen recycling via deconjugation by $\beta$-glucuronidase (GUS) from gut commensal microbes [9]. Intestinal microbial richness and enzymatic activity, including but not limited to GUS, have been shown to influence the levels of non-ovarian oestrogens through the enterohepatic circulation [10]. Indeed, high levels of oestrogens were observed to correlate to a high incidence of breast cancer [11]. Women with a more diverse gut microbiome have been 
shown to exhibit elevated urinary hydroxylated oestrogen metabolites [12].

During ER-independent mammary tumourigenesis, oestrogens are converted to 2-hydroxyestradiol (2-OHE2), 4-hydroxyestradiol (4-OHE2), semi-quinones, and quinones by cytochrome P450 enzymes, among which quinones can react with DNA to produce apurinic nucleotide and lead to A-T to G-C transition [13]. The highly depurinated adducts have also been detected in serum and urine samples from breast cancer patients and women with a strong family history of breast cancer [14]. Mouse gene mutations induced by 2-OHE2 and 4-OHE2 have been confirmed to cause uterine cancer [15] and kidney cancer [16]. The concentration of 4-OHE2 in breast cancer biopsies was found to be 3 times higher than in normal breast tissue [17], and urinary 2-OHE2 and 4-OHE2 levels were elevated in breast cancer patients compared with healthy controls [18].

Regarding the ER-dependent mammary tumourigenic mechanism, it remains unclear how can inflammation induce mammary hyperplasia. According to clinical data indicating a family-transmitted ER $\alpha$ mutation (R394H) with hyperoestrogenemia ( $>50$-fold normal range) [19], we anticipated that inactivation of ESR 1 -encoding ER $\alpha$ would result in oestrogen insensitivity and oestrogenemia. Because pro-inflammatory cytokines can activate NOS2-encoding inducible nitric oxide synthase (iNOS) for nitric oxide (NO) generation and peroxynitrite $\left(\mathrm{ONOO}^{-}\right)$formation, $\mathrm{NO}-$ and $\mathrm{ONOO}^{-}$-triggered $\mathrm{ER} \alpha$ nitrosylation can affect $\mathrm{ER} \alpha$ activity [20]. Therefore, iNOS activation and ER $\alpha$ inactivation should induce oestrogenemia and provoke mammary tumourigenesis or carcinogenesis.

Based on the above reasoning, we suggest herein a hypothesis of "breast cancer origin from iNOS activation and ER $\alpha$ inactivation" for deciphering ER-dependent mammary tumourigenesis. To verify this hypothesis, we intend to silence NOS2 and prohibit iNOS activation for reversing tumour progression, on the one hand, and to silence ESRI and mimic ER $\alpha$ inactivation for imitating tumour origin, on the other hand. Until now, no loss-of-function tests of NOS2 and ESR1 in tumour and normal cells, respectively, have been conducted to testify this putative tumour-inducing possibility.

Triple-negative breast cancer (TNBC), referring to any breast cancer that does not express ER, progesterone receptor (PR), and human epidermal growth factor 2 (HER2) receptor, is associated with a poor prognosis [21]. TNBC represents the female hormone analogue therapy-intractable subtype of breast cancer, accounting for $15 \%$ of clinical cases [22]. Using multivariate analysis, the new prognostic breast cancer marker $R U N X 1$ was identified to correlate with the poor prognosis specifically in TNBC [23]. By analysing the expressional profiles, it was found that $B C L 11 A$ is highly expressed in TNBC, 
and its overexpression in non-tumourigenic cells drives tumour development. In contrast, $B C L 11 A$ downregulation in TNBC cell lines leads to a reduction of tumour size [24].

To reverse or re-enact the expression and metabolic profiles of breast cancer, NOS2 or ESR1 was downregulated in the human TNBC cell line HCC1937 or the human mammary epithelial cell line MCF-10A. Additionally, E2 was supplemented in the latter to create a milieu mimicking the dysfunctional ESR1-induced oestrogenemia. We demonstrated for the first time that NOS 2 knockdown could convert breast cancer cells to nearly normal cells, whereas ESR1 knockdown could transform normal cells to breast cancer-like cells. This work, with prospects for an effective breast cancer therapy through the use of iNOS inhibitors and ER $\alpha$ activators, should shed light on the exploration of breast cancer origin, extend our scope of understanding regarding the mechanism underlying mammary tumourigenesis/carcinogenesis, and provide attractive and effective treatment regimens in hormone replacement-intractable TNBC patients.

\section{Methods}

\section{Cell culture, staining and counting}

The TNBC cell line HCC1937 (ATCC) was cultured in 1640 medium (Gibco) supplemented with $10 \%$ foetal bovine serum (FBS, Gibco). The mammary epithelial cell line MCF-10A (ATCC) over-expressing the recombinant ESR1 gene (LangRi, Guangzhou, China) was cultured in DMEM/F12 medium (Gibco) supplemented with 10\% FBS (Gibco). Cell thawing and subculture were conducted according to the standard procedures provided by the manufacturers. To plot the growth curve, live cells were stained with 3-(4,5-dimethyl-2-thiazolyl)-2,5-diphenyl-2-H-tetrazolium bromide (MTT), and the absorbance was measured at $490 \mathrm{~nm}$. To count the colony numbers, cells in the exponential growth phase were diluted to 500 cells $/ \mathrm{ml}$, cultured for 7-10 d, and stained with haematoxylin.

\section{Vector construction and stable transfection}

The retrovirus-based and integration-type plasmid expression vector pSuper-retro-puro (OligoEngine) was inserted separately into one of the NOS2- or ESR1-targeted shRNA fragments, siN1/2/3 or siE1/2/3, amplified using the primer pairs listed in Table 1 to obtain the recombinant constructs psiN1/2/3 or psiE1/2/3. After transient transfection and interference with efficiency identification, psiN1/2/3 or psiE1/2/3 was exploited to transfect HCC 1937 or MCF-10A for the establishment of a stable cell line. The procedures for restrictive digestion, ligation, transformation, and transfection were performed according to manufacturers' manuals.

\section{Table 1 Target sequence selection and primer design for NOS2IESR1}




\section{knockdown vector construction and transient expression.}

\section{A. Target choice and sequence alignment for NOS2 knockdown}

\begin{tabular}{|c|c|}
\hline Target/primer & Sequence \\
\hline Target 1 & 3'GGGAAAGAAGTCTCCAGAATC5' \\
\hline siN1 $\mathrm{F}$ & 5“GATCCCCGGGAAAGAAGTCTCCAGAATCTTCAAGAGAGATTCTGGAGACTTCTTTCCCTTTTTA3‘ \\
\hline siN1 R & 5'AGCTTAAAAAGGGAAAGAAGTCTCCAGAATCTCTCTTGAAGATTCTGGAGACTTCTTTCCCGGG3' \\
\hline Target 2 & 3'GCGTTACTCCACCAACAATGG5' \\
\hline siN2 F & 5‘GATCCCCGCGTTACTCCACCAACAATGGTTCAAGAGACCATTGTTGGTGGAGTAACGCTTTTTA3` \\
\hline siN2 R & 5'AGCTTAAAAAGCGTTACTCCACCAACAATGGTCTCTTGAACCATTGTTGGTGGAGTAACGCGGG3' \\
\hline Target 3 & 3'GCTCAAATCTCGGCAGAATCT5' \\
\hline siN3 F & 5‘GATCCCCGCTCAAATCTCGGCAGAATCTTTCAAGAGAAGATTCTGCCGAGATTTGAGCTTTTTA3‘ \\
\hline siN3 R & 5'AGCTTAAAAAGCTCAAATCTCGGCAGAATCTTCTCTTGAAAGATTCTGCCGAGATTTGAGCGGG3' \\
\hline siC F & 5'GATCCCCGCCAGCTTAGCACTGACTCTTCAAGAGAGAGTCAGTGCTAAGCTGGCTTTTTA3' \\
\hline siC R & 5’AGCTTAAAAAGCCAGCTTAGCACTGACTCTCTCTTGAAGAGTCAGTGCTAAGCTGGCGGG3' \\
\hline
\end{tabular}

\section{B. Target choice and sequence alignment for ESR1 knockdown}

\begin{tabular}{|c|c|}
\hline Target/primer & Sequence \\
\hline Target 1 & 3'GCATTCTACAGGCCAAATTCA5' \\
\hline siE1 F & 5‘GATCCCCGCATTCTACAGGCCAAATTCATTCAAGAGATGAATTTGGCCTGTAGAATGCTTTTTA3‘ \\
\hline SiE1 R & 5'AGCTTAAAAAGCATTCTACAGGCCAAATTCATCTCTTGAATGAATTTGGCCTGTAGAATGCGGG3' \\
\hline Target 2 & 3'GCTAGAGATCCTGATGATTGG5' \\
\hline siE2 F & 5‘GATCCCCGCTAGAGATCCTGATGATTGGTTCAAGAGACCAATCATCAGGATCTCTAGCTTTTTA3` \\
\hline SiE2 R & 5'AGCTTAAAAAGCTAGAGATCCTGATGATTGGTCTCTTGAACCAATCATCAGGATCTCTAGCGGG3' \\
\hline Target 3 & 3'GGGCTCTACTTCATCGCATTC5' \\
\hline siE3 F & 5‘GATCCCCGGGCTCTACTTCATCGCATTCTTCAAGAGAGAATGCGATGAAGTAGAGCCCTTTTTAA3 \\
\hline SiE3 R & 5'AGCTTAAAAAGGGCTCTACTTCATCGCATTCTCTCTTGAAGAATGCGATGAAGTAGAGCCCGGG3' \\
\hline siC F & 5“GATCCCC GCCAGCTTAGCACTGACTC TTCAAGAGA GAGTCAGTGCTAAGCTGGC TTTTT A3“ \\
\hline siC R & 5'AGCTT AAAAA GCCAGCTTAGCACTGACTC TCTCTTGAA GAGTCAGTGCTAAGCTGGC GGG $3^{\prime}$ \\
\hline
\end{tabular}

\section{Tumour cell grafting on nude mice}

BALB/c nude mice (5 weeks old) purchased from SKA, Changsha, China were housed with AL chow and free access to water drinking. For grafting, tumour cells were digested with trypsin, followed by centrifugation, re-suspension, and dilution to $1 \times 10^{5}$ cells $/ \mathrm{ml}$. The cell suspension $(100 \mu \mathrm{l})$ was subcutaneously injected into the hind legs of nude mice.

\section{Quantitative polymerase chain reaction (qPCR) and reverse transcription (RT)-PCR microarray}

RNA isolation, purity determination, electrophoresis monitoring, reverse transcription, and quantification were performed according to the standard 
protocol. The relative copy numbers $=2^{-\Delta \Delta \mathrm{C}_{\mathrm{T}}}$, where $\Delta \mathrm{CT}_{\mathrm{T}}=\mathrm{Ct}$ target gene $-\mathrm{Ct}$ reference gene and $\Delta \Delta \mathrm{C} \mathrm{T}=\Delta \mathrm{C}$ treatment sample $-\Delta \mathrm{C}$ control sample. The primers listed in Table 2 were designed and applied under the following amplification condition: $95^{\circ} \mathrm{C}, 60 \mathrm{~s}$; and $95^{\circ} \mathrm{C}, 30 \mathrm{~s}, 60^{\circ} \mathrm{C}, 35 \mathrm{~s}$ for 40 cycles. The RT $^{2}$ Profiler ${ }^{\mathrm{TM}}$ PCR Array Mouse Fatty Liver ( PAMM-157Z ) was purchased from SABioscience Qiagen, Hilden, Germany. The experiment was performed by Kangchen, Shanghai, China.

Table 2 Primer sequences for target gene amplification.

\begin{tabular}{|c|c|c|}
\hline Primer & Target gene & Sequence \\
\hline $\begin{array}{l}\text { H-SOD1-F-227 } \\
\text { H-SOD1-R-227 }\end{array}$ & $\begin{array}{l}\text { Copper, zinc-superoxide dismutase (Cu, } \\
\text { Zn-SOD) }\end{array}$ & $\begin{array}{l}\text { 5'GGTGGGCCAAAGGATGAAGAG3' } \\
\text { 5'CCACAAGCCAAACGACTTCC3' }\end{array}$ \\
\hline $\begin{array}{l}\mathrm{H}-\mathrm{IDH} 1-\mathrm{F}-147 \\
\mathrm{H}-\mathrm{IDH} 1-\mathrm{R}-147\end{array}$ & Isocitrate dehydrogenase 1 (IDH1) & $\begin{array}{l}\text { 5'TGTGGTAGAGATGCAAGGAGA3' } \\
\text { 5'TTGGTGACTTGGTCGTTGGTG3' }\end{array}$ \\
\hline $\begin{array}{l}\text { H-OGDH-F-81 } \\
\text { H-OGDH-R-81 }\end{array}$ & a-Ketoglutarate dehydrogenase (OGDH) & $\begin{array}{l}\text { 5'GGCTTCCCAGACTGTTAAGAC3' } \\
\text { 5'GCAGAATAGCACCGAATCTGTTG3' }\end{array}$ \\
\hline $\begin{array}{l}\text { H-DLST-F-79 } \\
\text { H-DLST-R-79 }\end{array}$ & $\begin{array}{l}\text { Dihydrolipoamide S-succinyltransferase } \\
\text { (DLST) }\end{array}$ & $\begin{array}{l}\text { 5'GAACTGCCCTCTAGGGAGAC3' } \\
\text { 5'AACCTTCCTGCTGTTAGGGTA3' }\end{array}$ \\
\hline $\begin{array}{l}\text { H-DLD-F-179 } \\
\text { H-DLD-R-179 }\end{array}$ & Dihydrolipoamide dehydrogenase (DLD) & $\begin{array}{l}\text { 5'CTCATGGCCTACAGGGACTTT3' } \\
\text { 5'GCATGTTCCACCAAGTGTTTCAT3' }\end{array}$ \\
\hline $\begin{array}{l}\text { H-PDHA1-F-151 } \\
\text { H-PDHA1-R-151 }\end{array}$ & $\begin{array}{l}\text { Pyruvate dehydrogenase (lipoamide) alpha } 1 \\
\text { (PDHA) }\end{array}$ & $\begin{array}{l}\text { 5'TGGTAGCATCCCGTAATTTTGC3' } \\
\text { 5'ATTCGGCGTACAGTCTGCATC3' }\end{array}$ \\
\hline $\begin{array}{l}\text { H-SOD2-F-116 } \\
\text { H-SOD2-R-116 }\end{array}$ & $\begin{array}{l}\text { Manganese superoxide dismutase } \\
\text { (Mn-SOD) }\end{array}$ & $\begin{array}{l}\text { 5'GGAAGCCATCAAACGTGACTT3' } \\
\text { 5'CCCGTTCCTTATTGAAACCAAGC3' }\end{array}$ \\
\hline $\begin{array}{l}\text { H-CAT-F-209 } \\
\text { H-CAT-R-209 }\end{array}$ & Catalase (CAT) & $\begin{array}{l}\text { 5'TGGAGCTGGTAACCCAGTAGG3' } \\
\text { 5'CCTTTGCCTTGGAGTATTTGGTA3' }\end{array}$ \\
\hline $\begin{array}{l}\text { H-PDK1-F-191 } \\
\text { H-PDK1-R-191 }\end{array}$ & Pyruvate dehydrogenase kinase 1 (PDK1) & $\begin{array}{l}\text { 5'CTGTGATACGGATCAGAAACCG3' } \\
\text { 5'TCCACCAAACAATAAAGAGTGCT3' }\end{array}$ \\
\hline $\begin{array}{l}\text { H-PDP1-F-77 } \\
\text { H-PDP1-R-77 }\end{array}$ & $\begin{array}{l}\text { Pyruvate dehydrogenase phosphatase } \\
\text { catalytic subunit } 1 \text { (PDP1) }\end{array}$ & $\begin{array}{l}\text { 5'TGTGAACTGAGCAGGATCTATGG3' } \\
\text { 5'GGAATGTACGATGAGGAACAACA3' }\end{array}$ \\
\hline $\begin{array}{l}\text { H-LDHA-F-91 } \\
\text { H-LDHA-R-91 }\end{array}$ & Lactate dehydrogenase 1 (LDH1) & $\begin{array}{l}\text { 5'TTGACCTACGTGGCTTGGAAG3' } \\
\text { 5'GGTAACGGAATCGGGCTGAAT3' }\end{array}$ \\
\hline $\begin{array}{l}\text { H-NOS3-F } \\
\text { H-NOS3-R }\end{array}$ & Endothelial nitric oxide synthase (eNOS) & $\begin{array}{l}\text { 5'TGATGGCGAAGCGAGTGAAG3' } \\
\text { 5'ACTCATCCATACACAGGACCC3' }\end{array}$ \\
\hline $\begin{array}{l}\text { H-EPO-F } \\
\text { H-EPO-R }\end{array}$ & Erythropoietin (EPO) & $\begin{array}{l}\text { 5'GGAGGCCGAGAATATCACGAC3' } \\
\text { 5'CCCTGCCAGACTTCTACGG3' }\end{array}$ \\
\hline
\end{tabular}




\begin{tabular}{|c|c|c|}
\hline $\begin{array}{l}\text { H-SIRT1-F } \\
\text { H-SIRT1-R }\end{array}$ & Sirtuin type1 (SIRT1) & $\begin{array}{l}\text { 5'TAGCCTTGTCAGATAAGGAAGGA3' } \\
\text { 5'ACAGCTTCACAGTCAACTTTGT3' }\end{array}$ \\
\hline $\begin{array}{l}\text { H-NOS2-F } \\
\text { H-NOS2-R }\end{array}$ & Inducible nitric oxide synthase (iNOS) & $\begin{array}{l}\text { 5'AGGGACAAGCCTACCCCTC3' } \\
\text { 5'CTCATCTCCСGTCAGTTGGT3' }\end{array}$ \\
\hline $\begin{array}{l}\text { H-ERa-F } \\
\text { H-ERa-R }\end{array}$ & Oestrogen receptor $\alpha(E R \alpha)$ & $\begin{array}{l}\text { 5'GGGAAGTATGGCTATGGAATCTG3' } \\
\text { 5'TGGCTGGACACATATAGTCGTT3' }\end{array}$ \\
\hline 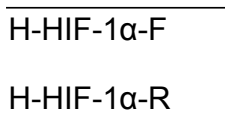 & Hypoxia inducible factor $\alpha$ (HIF-1 $1 \alpha)$ & $\begin{array}{l}\text { 5'ATCCATGTGACCATGAGGAAATG3' } \\
\text { 5'TCGGCTAGTTAGGGTACACTTC3' }\end{array}$ \\
\hline $\begin{array}{l}\mathrm{H}-\mathrm{HO}-1-\mathrm{F} \\
\mathrm{H}-\mathrm{HO}-1-\mathrm{R}\end{array}$ & Heme oxygenase 1 (HO-1) & $\begin{array}{l}\text { 5'AAGACTGCGTTCCTGCTCAAC3' } \\
\text { 5'AAAGCCCTACAGCAACTGTCG3' }\end{array}$ \\
\hline $\begin{array}{l}\text { H-VEGF-F } \\
\text { H-VEGF-R }\end{array}$ & Vascular epithelial growth factor (VEGF) & $\begin{array}{l}\text { 5'AGGGCAGAATCATCACGAAGT3' } \\
\text { 5'AGGGTCTCGATTGGATGGCA3' }\end{array}$ \\
\hline $\begin{array}{l}\mathrm{H}-\mathrm{COX} 4-\mathrm{F} \\
\mathrm{H}-\mathrm{COX} 4-\mathrm{R}\end{array}$ & Cytochrome c oxidase 4 (COX4) & $\begin{array}{l}\text { 5'CAGGGTATTTAGCCTAGTTGGC3' } \\
\text { 5'GCCGATCCATATAAGCTGGGA3' }\end{array}$ \\
\hline $\begin{array}{l}\text { H-AKT-F } \\
\text { H-AKT-R }\end{array}$ & $\begin{array}{l}\text { RAC-alpha serine/threonine-protein kinase } \\
\text { (Akt) }\end{array}$ & $\begin{array}{l}\text { 5'GTCATCGAACGCACCTTCCAT3' } \\
\text { 5'AGCTTCAGGTACTCAAACTCGT3' }\end{array}$ \\
\hline $\begin{array}{l}\text { H-AMPK-F } \\
\text { H-AMPK-R }\end{array}$ & $\begin{array}{l}\text { Adenosine } 5 \text { '-monophosphate-activated } \\
\text { protein kinase (AMPK) }\end{array}$ & $\begin{array}{l}\text { 5'TTGAAACCTGAAAATGTCCTGCT3' } \\
\text { 5'GGTGAGCCACAACTTGTTCTT3' }\end{array}$ \\
\hline $\begin{array}{l}\text { H-FOXO3a-F } \\
\text { H-FOXO3a-R }\end{array}$ & Forkhead box D3 (FOXO3A) & $\begin{array}{l}\text { 5'CGGACAAACGGCTCACTCT3' } \\
\text { 5'GGACCCGCATGAATCGACTAT3' }\end{array}$ \\
\hline 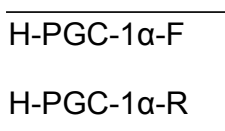 & $\begin{array}{l}\text { Peroxisome proliferator-activated receptor } \\
\text { gamma coactivator } 1 \text {-alpha (PGC-1 } \alpha)\end{array}$ & $\begin{array}{l}\text { 5'GCTTTCTGGGTGGACTCAAGT3' } \\
\text { 5'GAGGGCAATCCGTCTTCATCC3' }\end{array}$ \\
\hline $\begin{array}{l}\mathrm{H}-\mathrm{CD} 1-\mathrm{F} \\
\mathrm{H}-\mathrm{CD} 1-\mathrm{R}\end{array}$ & Cyclin D1 (CD1) & $\begin{array}{l}\text { 5'GCTGCGAAGTGGAAACCATC3' } \\
\text { 5'CCTCСTTCTGCACACATTTGAA3' }\end{array}$ \\
\hline $\begin{array}{l}\text { H-p21-F } \\
\text { H-p21-R }\end{array}$ & Cyclin-dependent kinase inhibitor 1 (CKI) & $\begin{array}{l}\text { 5'TGTCCGTCAGAACCCATGC3' } \\
\text { 5'AAAGTCGAAGTTCCATCGCTC3' }\end{array}$ \\
\hline $\begin{array}{l}\text { H-TP53BP1-F } \\
\text { H-TP53BP1-R }\end{array}$ & $\begin{array}{l}\text { Tumour suppressor p53-binding protein } 1 \\
\text { (TP53BP1) }\end{array}$ & $\begin{array}{l}\text { 5'CTCCAGACGCACAAAGAAAATCC3' } \\
\text { 5'ACCTGACTGATGGAACCACAT3' }\end{array}$ \\
\hline $\begin{array}{l}\text { H-ESRRG-F } \\
\text { H-ESRRG-R }\end{array}$ & $\begin{array}{l}\text { Oestrogen-related receptor gamma } \\
\text { (ESRRG) }\end{array}$ & $\begin{array}{l}\text { 5'GCCCTCACTACACTGTGTGAC3' } \\
\text { 5'CCTGCTAATTTGGACTGGTCTT3' }\end{array}$ \\
\hline $\begin{array}{l}\text { H-EPAS1-F } \\
\text { H-EPAS1-R }\end{array}$ & $\begin{array}{l}\text { Endothelial PAS domain-containing protein } 1 \\
\text { (EPAS1) }\end{array}$ & $\begin{array}{l}\text { 5'TTGATGTGGAAACGGATGAA3' } \\
\text { 5'GGAACCTGCTCTTGCTGTTC3' }\end{array}$ \\
\hline $\begin{array}{l}\text { H-BCL11A-F } \\
\text { H-BCL11A-R }\end{array}$ & B-cell lymphoma/leukaemia 11A (BCL11A) & $\begin{array}{l}\text { 5'CGCCAGAGGATGACGATTGTT3' } \\
\text { 5'CCAGGCGTGGGGATTAGAG3' }\end{array}$ \\
\hline
\end{tabular}




\begin{tabular}{lll}
\hline H-RUNX1-F & Runt-related transcription factor 1 (RUNX1) & 5'CCACTCCACTGCCTTTAACC3' \\
H-RUNX1-R & 5'CTGGGTGCACAGAAGGAGAG3' \\
\hline H-ATR-F & $\begin{array}{l}\text { Ataxia telangiectasia and Rad3-related } \\
\text { protein (ATR) }\end{array}$ & 5'GGCCAAAGGCAGTTGTATTGA3' \\
H-ATR-R & 5'GTGAGTACCCCAAAAATAGCAGG3' \\
\hline H-ESR2-F & Oestrogen receptor beta (ERß) & 5'CATGATCCTGCTCAATTCCA3' \\
H-ESR2-R & & 5'ACCAAAGCATCGGTCACG3' \\
\hline H-CYP1A1-F & $\begin{array}{l}\text { Cytochrome P450, family 1, subfamily A, } \\
\text { polypeptide 1 (CYP1A1) }\end{array}$ & 5'TCGGCCACGGAGTTTCTTC3' \\
H-CYP1A1-R & 5'GGTCAGCATGTGCCCAATCA3' \\
\hline
\end{tabular}

\section{Western blotting (WB) and enzyme-linked immunosorbent assay (ELISA)}

The reference protein glyceraldehyde-3-phosphate dehydrogenase (GAPDH) and antigen proteins were immunoquantified by WB according to the manufacturers' manuals. Antibodies, including anti-PGC-1 $\alpha$, anti-VEGF, anti-CD1, anti-AMPK $\alpha 1+\mathrm{AMPK} \alpha 2$, anti-AMPK $\alpha 1$ (phospho T183)+AMPKa2 (phospho T172), anti-pan-Akt, anti-pan-Akt (phospho T308), anti-eNOS, anti-iNOS, anti- $\alpha$-tubulin, anti-HIF- $1 \alpha$, anti-HO-1, anti-EPO, anti-COX4, anti-CKI/p21, anti-SIRT1, anti-FOXO3A, anti-FOXO3A (phospho S253), and anti-Ki67 (PCNA), were purchased from Abcam (Shanghai, China). Anti-E-cadherin was provided by BOSTER (Wuhan, China), and anti-mTOR was provided by CST (Shanghai, China).

\section{Histochemical and immunohistochemical analysis}

Formalin fixation, paraffin embedding and deparaffinization were performed as described for the haematoxylin and eosin (HE) staining procedure. Sections were incubated at room temperature with $3 \% \mathrm{H}_{2} \mathrm{O}_{2}$ to block endogenous peroxidase and then repaired in boiling citric acid. After washing in phosphate-buffered solution, the sections were blocked with $2 \%$ bovine serum albumin and incubated with the $1: 100$ diluted primary antibodies at $37^{\circ} \mathrm{C}$ for 1 $\mathrm{h}$. The primary antibody against 3-nitrotyrosine (NT) was purchased from Abcam. After washing, the sections were incubated with biotinylated secondary antibodies at $37^{\circ} \mathrm{C}$ for $20 \mathrm{~min}$. After washing again, the sections were incubated with diaminobenzidine for 1-5 min. After rinsing with tap water, the sections were counter-stained with haematoxylin. After completion of dehydration, clearance and mounting, images were obtained under an OLYMPUS BX-51 microscope.

\section{Electronic microscopy}

After treatment, the cells were harvested and fixed in $2.5 \%$ glutaraldehyde in $0.1 \mathrm{M}$ phosphate buffer for three hours at $4^{\circ} \mathrm{C}$, followed by post-fixation in $1 \%$ osmium tetroxide for one hour. Samples were dehydrated in a graded 
series of ethanol baths and infiltrated and embedded in Spurr's low-viscosity medium. Ultra-thin sections of $60 \mathrm{nM}$ were cut in a Leica microtome, double-stained with uranyl acetate and lead acetate, and examined using a Hitachi 7700 transmission electron microscope at an accelerating voltage of $60 \mathrm{kV}$.

\section{Apoptosis and reactive oxygen species (ROS) detection}

The Annexin V-fluorescein isothiocyanate (FITC) Apoptosis Detection Kit was purchased from Sigma-Aldrich (USA). Cells were suspended in binding buffer and mixed with Annexin V-FITC and propidium iodide (PI) for the cytometric assay. The FITC-labelled TdT-mediated dUTP nick-end labelling (TUNEL) apoptosis detection kit was purchased from Keygen (Nanjing, China). Cells were incubated with the TdT enzyme reaction solution for fluorescence microscopic analysis. The ROS detection kit was purchased from Beyotime (Guangzhou, China). Cells were incubated with the diluted dichlorodihydrofluorescein diacetate (DCFH-DA) for detection using the fluorescence microplate reader at a wave length of 488/525 nm.

\section{Chorioallantoic membrane tests}

The one-week-old embryonated egg was drilled to place a piece of sterilised filter paper on the 0.5 -cm-diameter chorioallantoic membrane surface, and then it was loaded with $200 \mu$ of the conditional culture medium suspension containing HCC1937::psiC or HCC1937::psiN3 cells. After sealing and incubation at $37^{\circ} \mathrm{C}$ with a humidity of $80 \%$ for $48-72 \mathrm{~h}$, the chorioallantoic membrane was sheared to obtain images.

\section{Spectrophotometry}

The lactic acid (LA) and adenosine triphosphate (ATP) reagent kits were purchased from Jiancheng Biotech, Nanjing, China. All determination procedures were conducted according to the manufacturers' instructions.

\section{Statistical analysis}

The software SPSS 22.0 was employed to analyse the raw data, and the software GraphPad Prism 5.0 was employed to plot the graphs. The independent sample test was used to compare all groups, and the Kruskal-Wallis test followed by the Nemenyi test was used when the data distribution was skewed. The significance level ( $\mathrm{p}$ value) was set at $<0.05$ (*) $^{*}$ $<0.01(* *),<0.001(* * *)$ and $<0.0001(* * * *)$.

\section{Results}

NOS2 knockdown results in a decline in iNOS and expression level changes of oncogenes, tumour suppressor genes and TNBC-specific transcription factor genes 
Three recombinant NOS2-targeted small interfering RNA (siRNA) expression constructs, psiRNA-NOS1/2/3 (abbr. psiN1/2/3), were available by inserting one of three candidate shRNA fragments (see the sequence alignment in Methods), NOS 2 shRNA1/2/3, into the integration-type plasmid expression vector pSUPER-retro-puro. After separately transferring these vehicles into HCC1937 cells, NOS2 mRNA and iNOS levels were quantified in HCC1937::psiN1/2/3 cells transiently expressing NOS2 shRNA.

Compared with the control HCC1937::psiC cells, NOS2 mRNAs and iNOS levels declined to different extents in HCC1937::psiN1/2/3 cells (Fig. 1a and $1 b)$. The expression profiles showed that psiN3 exhibited a high efficiency for interfering with NOS2 expression among others, so psiN3 was chosen to stably transfect HCC1937 cells to gain HCC1937::psiN3 cells with permanent NOS2 shRNA expression.

By monitoring the dynamic changes in tumourigenesis-related markers upon targeted gene knockdown, we observed that the expression levels of tumour marker CD1-encoded mRNA levels declined, whereas tumour suppressor CKI/P21-encoded mRNA levels were elevated in HCC1937::psiN3 cells (Fig. 1c). Similarly, while CD1 levels declined, CKI levels exhibited an elevation in HCC1937::psiN3 cells (Fig. 1d).

By quantifying the TNBC-specific transcription factors $R U N X 1$ and $B C L 11 A$ mRNA levels, we noticed that NOS2 knockdown could markedly downregulate $R U N X 1$ and $B C L 11 A$ expression $(P<0.0001)$ (Fig. 1e), suggesting that NOS 2 activated by pro-inflammatory cytokines might represent a pivotal step toward breast cancer.

a

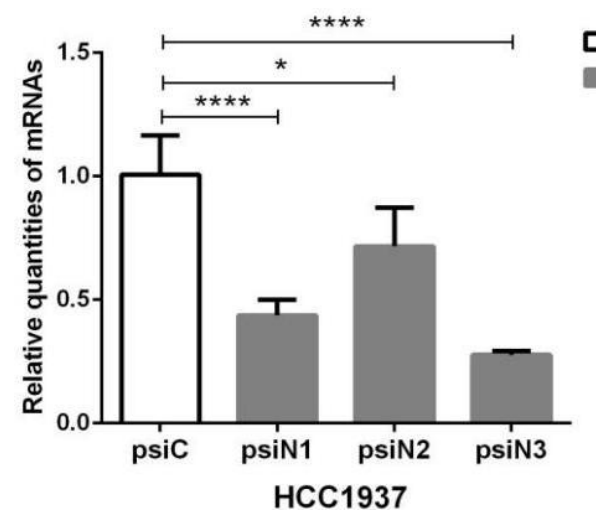

b

HCC1937

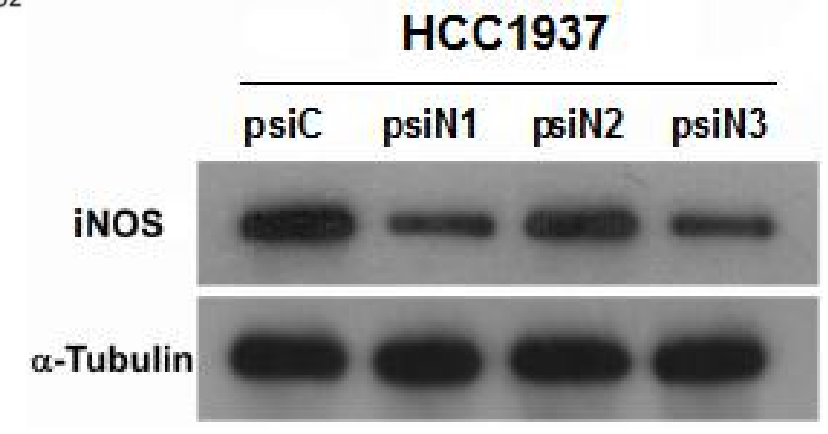


C

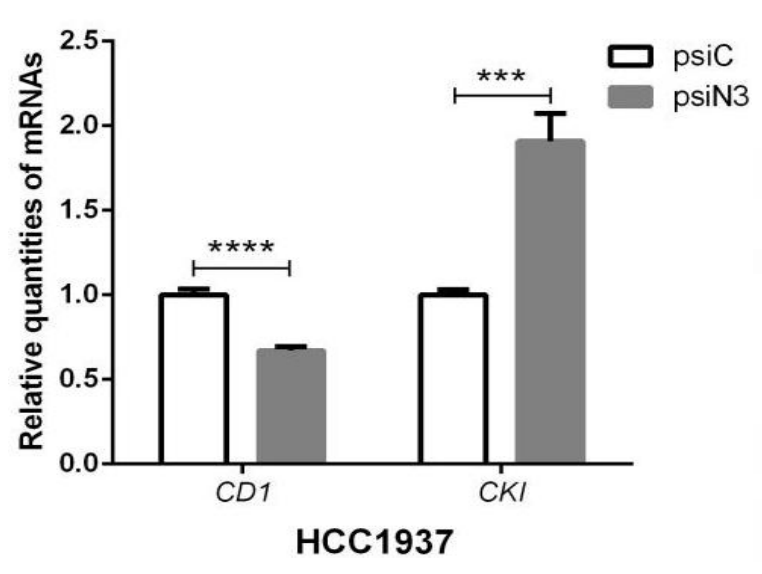

d

HCC1937

CD1 psiC psiN3

CKI

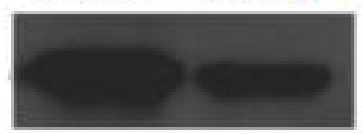

$\alpha$-tubulin

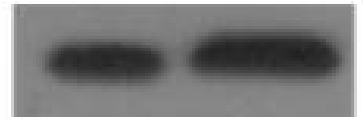

e

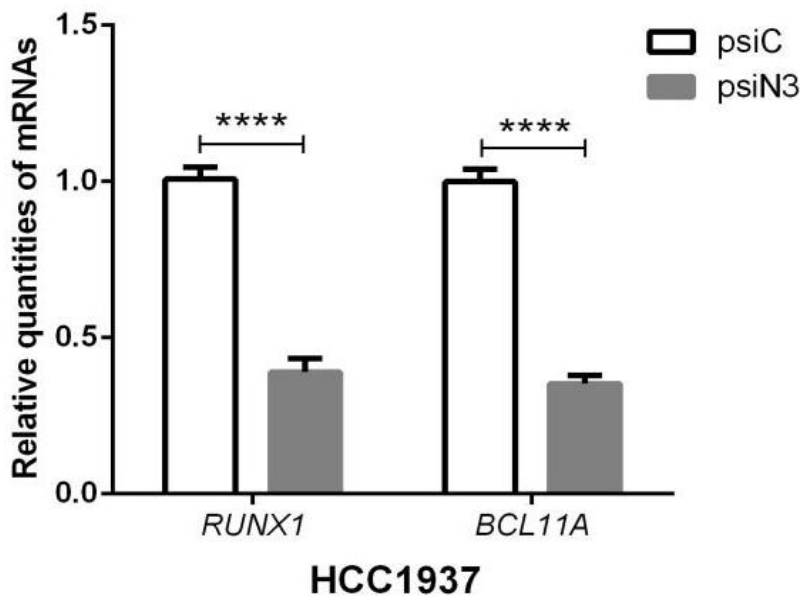

Fig. 1

The expression profiles of NOS2 mRNA/iNOS, CD1 mRNA/CD1, CKI mRNA/CKI, RUNX1 mRNA and BCL11A mRNA after shRNA-guided NOS2 knockdown in HCC1937::psiC/psiN1/2/3 cells. a NOS2 mRNA levels (transient expression); b iNOS levels (transient expression); c CD1 and CKI mRNA levels; $\mathbf{d} C D 1$ and CKI levels; e RUNX1 and BCL11A mRNA levels.

NOS2 downregulation renders NOS3 upregulation with a high level of NO but a low level of ROS that unalters nitrosylation and apoptosis

To assess the impact of NOS2 knockdown on the correlation of NOS2/ iNOS with NOS3/eNOS, we quantified the NOS2 and NOS3 mRNA levels (Fig. 2a) as well as the iNOS/eNOS levels (Fig. 2b) in HCC1937::psiC/psiN3 cells. As noted, the decline in NOS2 mRNA and iNOS levels was accompanied by an elevation of NOS3 mRNA and eNOS levels. Accordingly, an elevated NO level (Fig. 2c) was correlated with a decline in the ROS level (Fig. 2d). At a low level of ROS, ROS-scavenging SOD and CAT levels both declined (Fig. 2e and 2f).

Because of low-level ROS, the ROS-NO reaction-derived product ONOOshould decrease accordingly. Therefore, the green fluorescence-labelled proteins carrying the $\mathrm{ONOO}^{-}$-modifying tyrosine residue 3-nitrotyrosine (NT) 
were almost equivalent between HCC1937::psiC and HCC1937::psiN3 cells (Fig. 2g). As an essential outcome, the early-phase and late-phase apoptotic cell percentages were also identical compared HCC1937::psiC cells with HCC1937::psiN3 cells (5.0\% versus 4.4\%) (Fig. 2h).

a

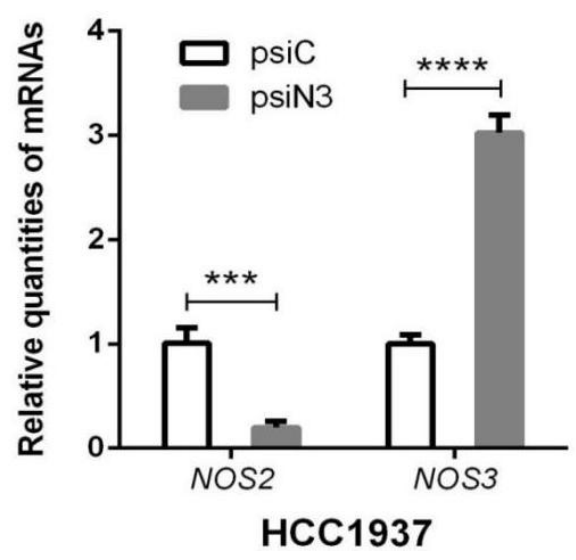

C

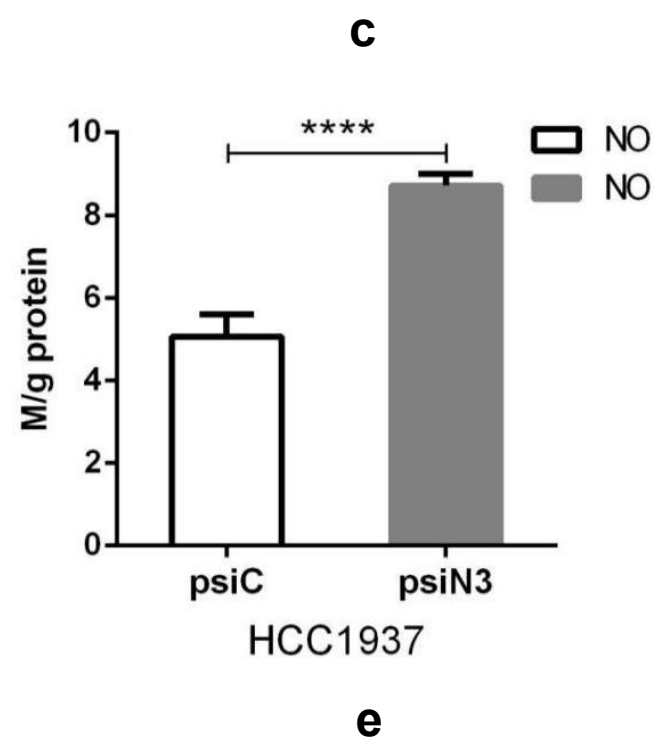

b

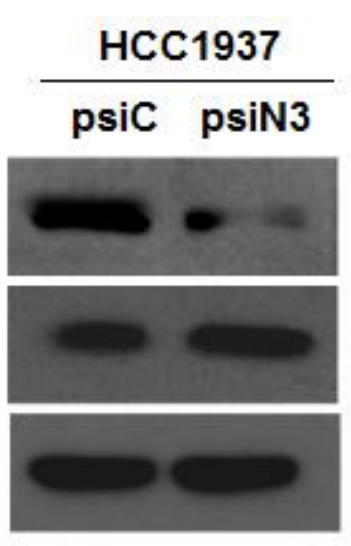

d
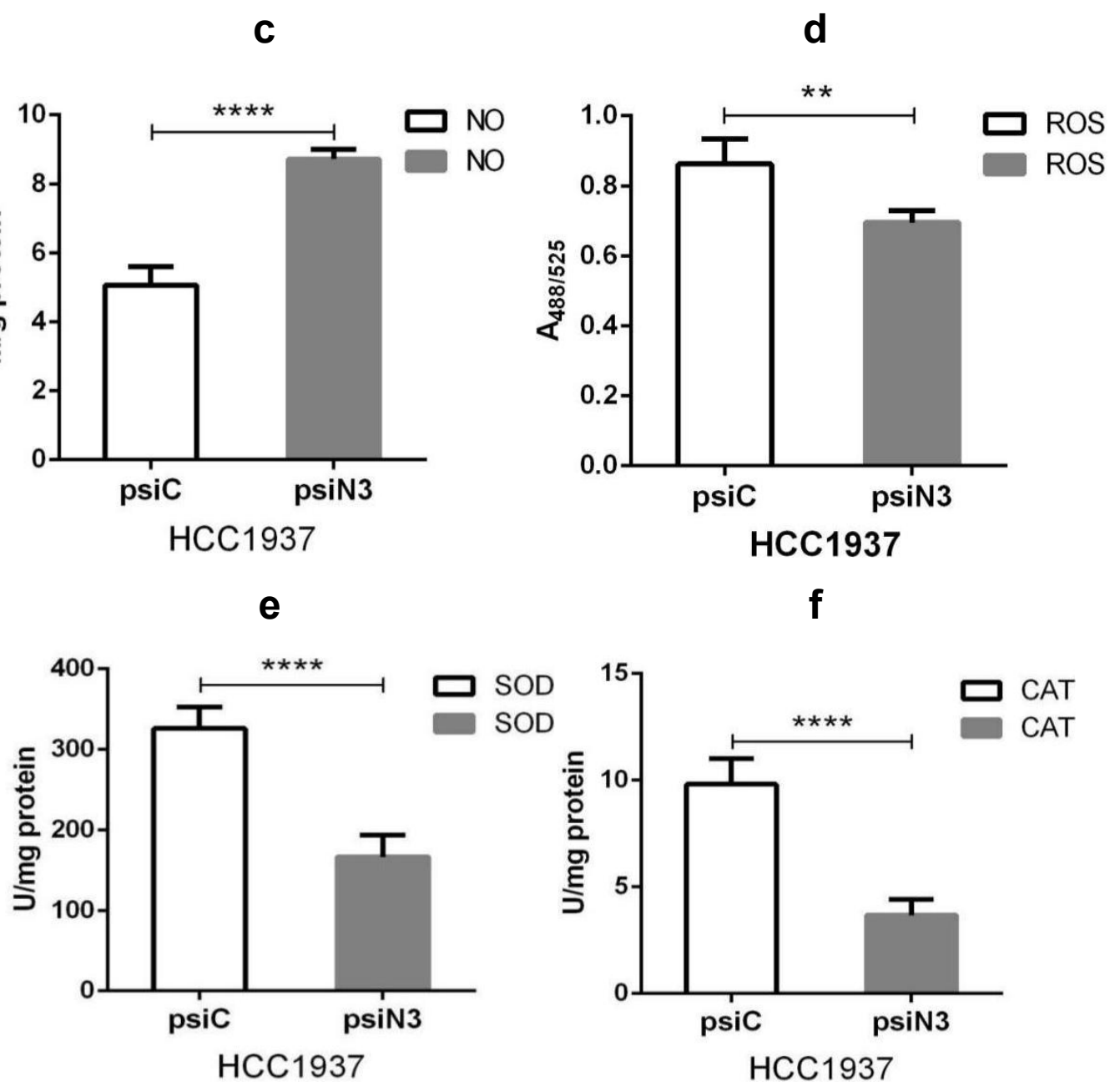


\section{g}

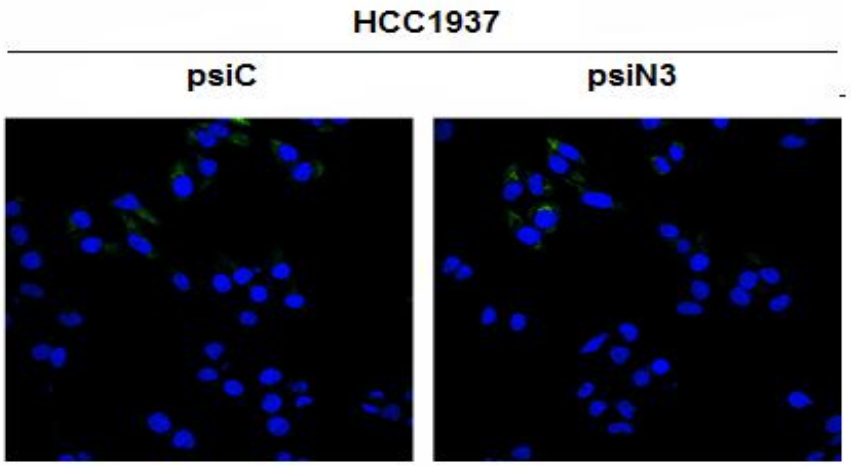

h

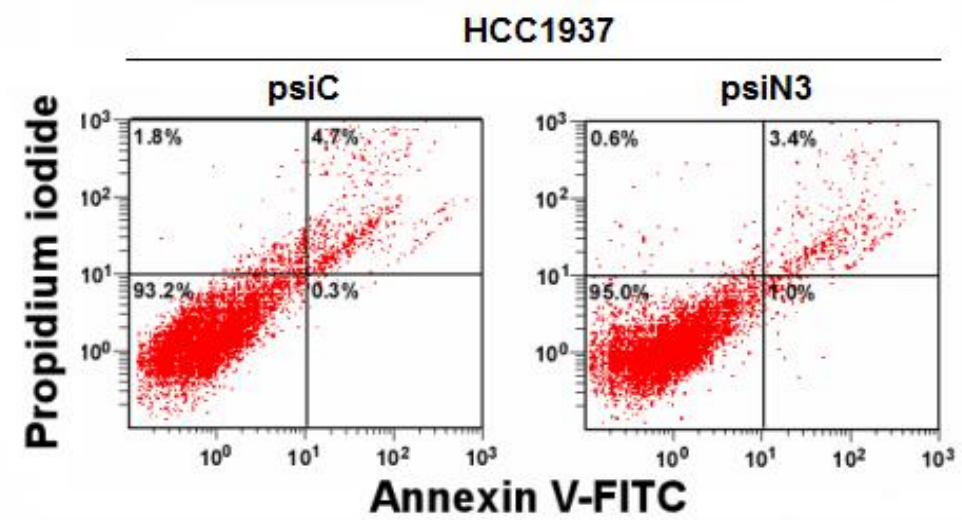

Fig. 2

Effects of NOS2 knockdown on NOS2 mRNA/iNOS, NOS3 mRNA/eNOS, NO, ROS, SOD, CAT levels, nitrosylation and apoptosis in HCC1937::psiC/psiN3 cells. a NOS2 and NOS3 mRNA levels; b iNOS and eNOS levels; c NO levels; $\boldsymbol{d}$ ROS levels. e SOD levels; $\mathbf{f}$ CAT levels; $\mathbf{g}$ NT levels in HCC1937::psiC cells (left) and HCC1937::psiN3 cells (right); $\mathbf{h}$ apoptosis percentages in HCC1937::psiC cells (left) and HCC1937::psiN3 cells (right).

\section{An attenuated Warburg effect correlates with accelerated glucose transport into mitochondria for Krebs cycle reactions}

To evaluate whether NOS2 knockdown would affect the metabolic patterns of tumour cells, we determined the levels of ATP, an aerobic glucose catabolic end product, and LA, an anaerobic glucose catabolic end product, in HCC1937::psiC/psiN3 cells. As a result, while ATP increased (Fig. 3a), LA decreased (Fig. 3b), suggesting a metabolic conversion from glycolysis to Krebs cycle/citric acid cycle reactions, i.e., the Warburg effect was attenuated upon NOS2 knockdown in TNBC cells.

To further determine how anaerobic catabolism could be converted to aerobic catabolism, we quantified the levels of mRNAs encoding the critical substrate transporter and Krebs cycle enzymes in HCC1937::psiC/psiN3 cells. As illustrated in Fig. 3c, the mRNA levels of glucose transporter 1 (GLUT1) responsible for glucose intake into cells was nearly unchanged, whereas that of monocarboxylate transporter 4 (MCT4) responsible for the transport of 
pyruvate, an intermediate of aerobic glucose catabolism, into mitochondria was markedly increased, suggesting the complete degradation of glucose via pyruvate in mitochondria. Accordingly, the mRNA of hexokinase 2 (HK2) mRNA, which encodes an enzyme that degrades glucose to pyruvate, and 6-phosphofructokinase liver type (PFKL) and isocitrate dehydrogenase 1 (IDH1), which encode the enzymes that degrade pyruvate, were also significantly increased; however, the mRNAs of the other enzyme-encoded oxoglutarate dehydrogenase $(O G D H)$ and dihydrolipoamide S-succinyltransferase $(D L S T)$ were upregulated without statistical significance (Fig. 3c).

In the pivotal controlling centre determining glycolysis or Krebs cycle reactions, the pyruvate dehydrogenase complex (PDC), pyruvate dehydrogenase alpha 1 (PDHA1)-encoded mRNA was significantly elevated, although dihydrolipoyl dehydrogenase (DLD)-encoded mRNA remained unchanged. While the mRNA encoding pyruvate dehydrogenase kinase isozyme 1 (PDK1) for PDH inactivation by phosphorylation was increased without statistical significance, the mRNA encoding pyruvate hydrogenase phosphatase 1 (PDP1) for PDH activation by dephosphorylation was increased with statistical significance $(P<0.05)$. Nevertheless, lactic acid dehydrogenase A (LDHA) was still upregulated, suggesting that the conversion from glucose to LA via glycolysis was partially maintained (Fig. 3d). As evidence confirming the co-existence of anaerobic and aerobic glucose catabolism, both PDK1 that inactivates PDH and PDP1 that activates PDH were synchronously increased (Fig. 3e).

a

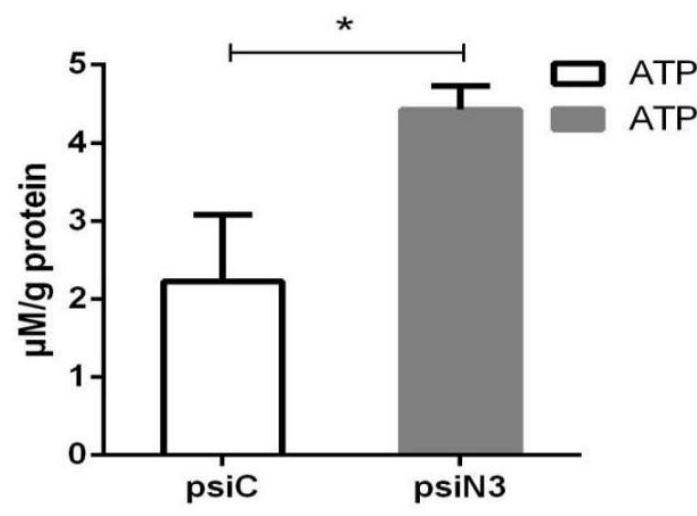

HCC1937 b

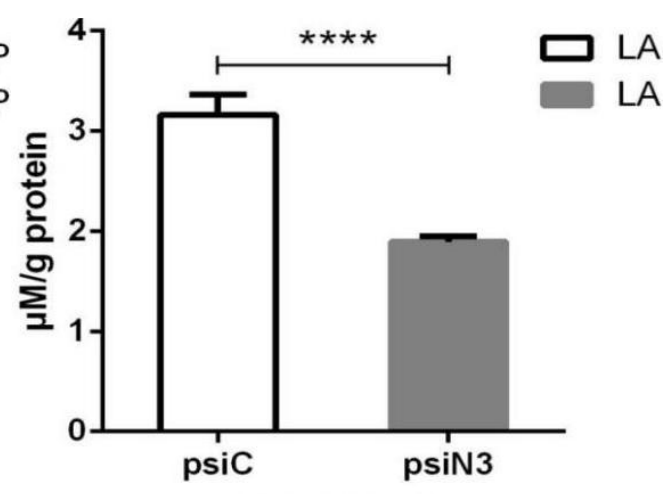

HCC1937 

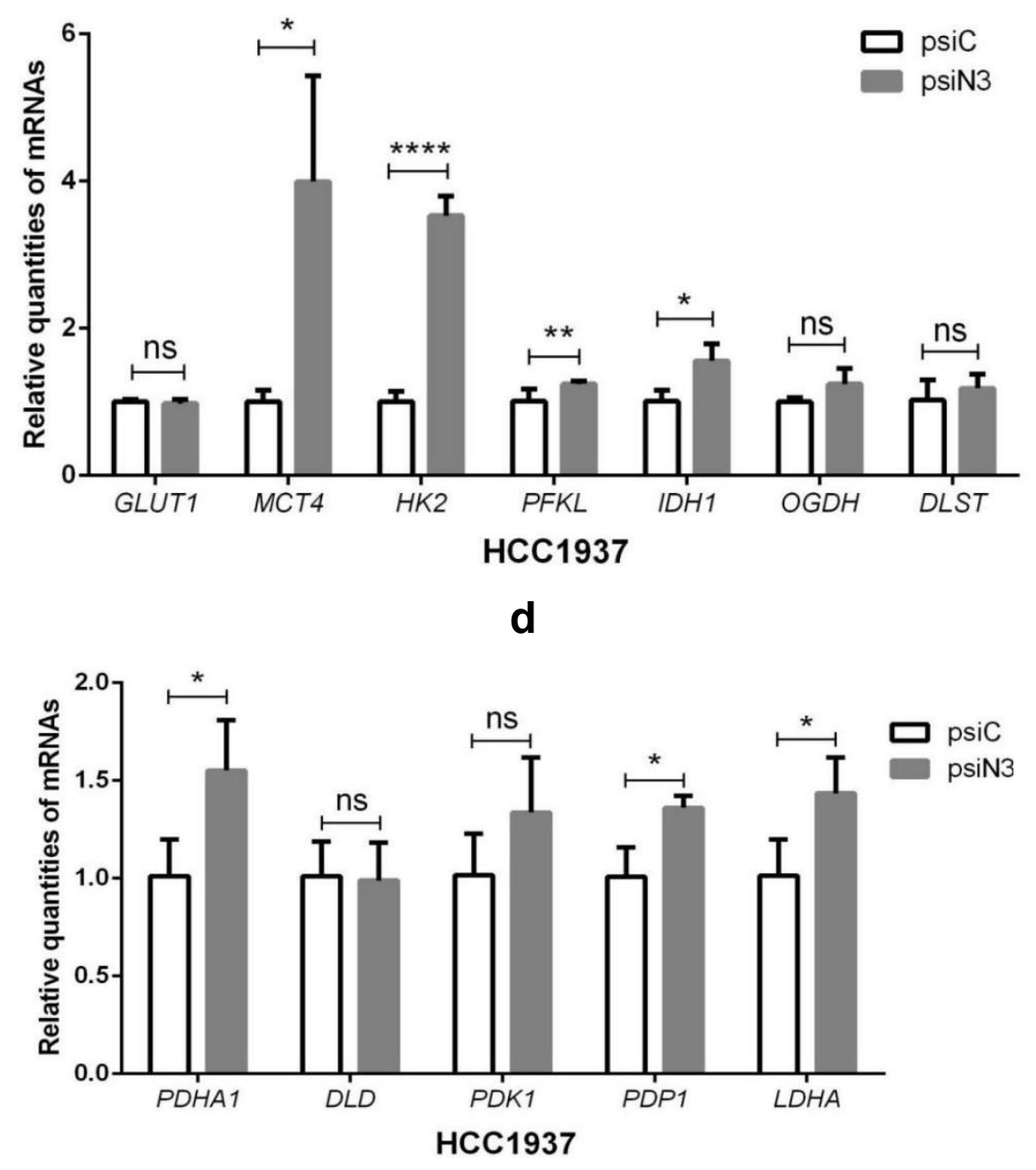

e

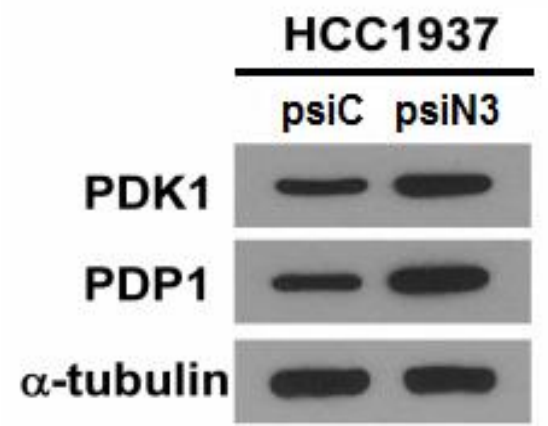

Fig. 3

Impact of NOS2 knockdown on glycolytic intermediate transport and aerobic/anaerobic glucose degradation in HCC1937::psiC/psiN3 mice. a ATP levels; b LA levels; c GLUT1, MCT4, HK2, PFKL, IDH1, OGDH, and DLST mRNA levels; d PDHA1, DLD, PDK1, PDP1, and LDHA mRNA levels; e PDK1 and PDP1 levels.

Enhanced AMPK/SIRT1/PGC-1a signalling cascades drive mitochondrial biogenesis but unchange angiogenesis

To evaluate the effect of NOS 2 knockdown on mitochondrial biogenesis, we 
quantified the expression levels of AMPK/SIRT1/PGC-1 $\alpha$ signal transducers in HCC1937::psiC/psiN3 cells. As a result, $A M P K(p<0.05), \operatorname{SIRT1}(p<0.05)$, $P G C-1 \alpha(p<0.01)$ and COX4 mRNAs $(p<0.05)$ were synchronously increased with statistical significance (Fig. 4a), although AMPK, pAMPK, Akt, pAkt, SIRT1, PGC-1 $\alpha$, and COX4 were only slightly increased (Fig. 4c).

To explore whether NOS2 knockdown might affect hypoxia-inducible genes, we quantified the expression levels of HIF-1 $\alpha$ and some downstream genes in HCC1937::psiC/psiN3 cells. Excluding HIF-1 $\alpha$ mRNA, HO-1 mRNA $(p<0.01)$ for anti-oxidation, EPO mRNA $(p<0.05)$ for erythrogenesis, and $V E G F$ mRNA $(p<0.05)$ for angiogenesis were overexpressed upon NOS2 knockdown (Fig. 4b). However, HIF-1 $\alpha$ maintained a constant level despite the elevation of HO-1, EPO and VEGF (Fig. 4d), implying that HIF-1 $\alpha$ was subjected to post-translational regulation. While the tumour suppressor-encoding FoxO3a mRNA increased insignificantly, FOXO3A and p-FOXO3A are increased with discernible differences.

As shown, the mitochondria were larger but scarce in HCC1937::psiC cells, whereas they were smaller but extensively distributed in HCC1937::psiN3 cells (Fig. 4e), indicating that NOS2 knockdown might have driven mitochondrial biogenesis by activating AMPK/SIRT1/PGC-1 $\alpha$ signalling. Additionally, no distinguishable difference in angiogenesis phenotypes was found in chick chorioallantoic membrane treated with HCC1937::psiN3 cells compared with HCC1937::psiC cells, albeit the presence of more blood knots following the treatment with HCC1937::psiN3 cells (Fig. 4f).

a

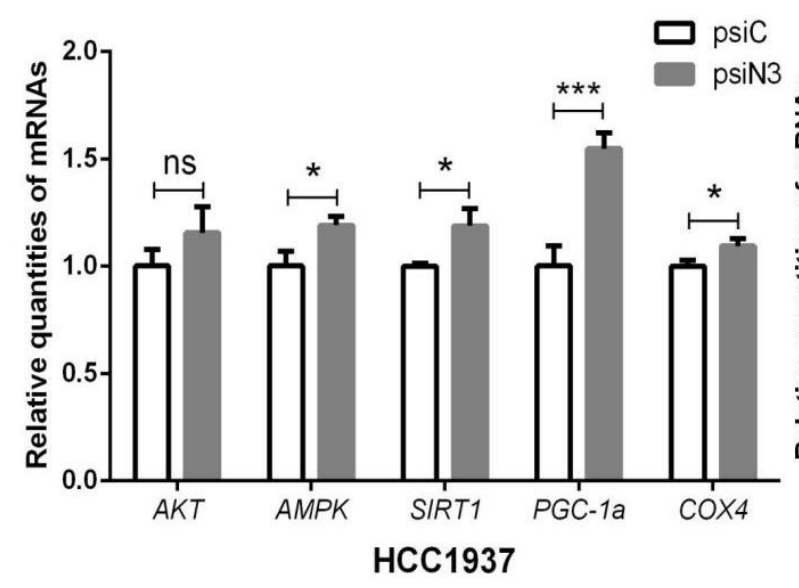

b

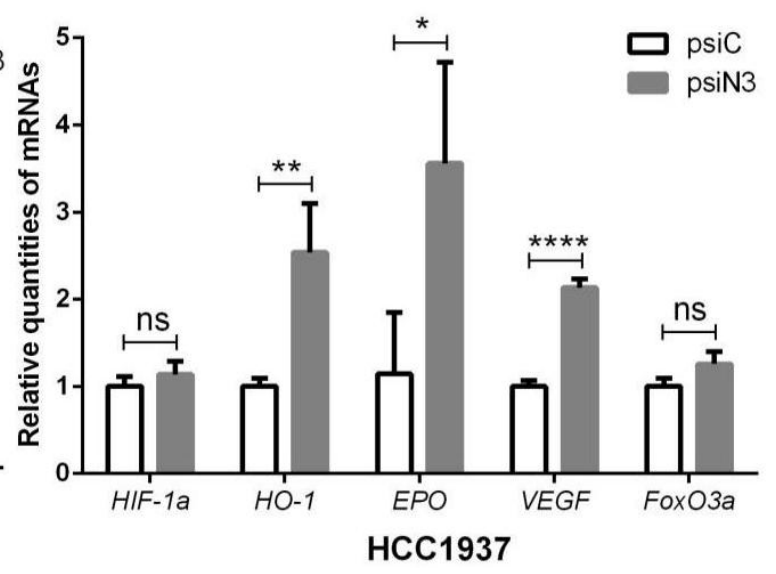


C

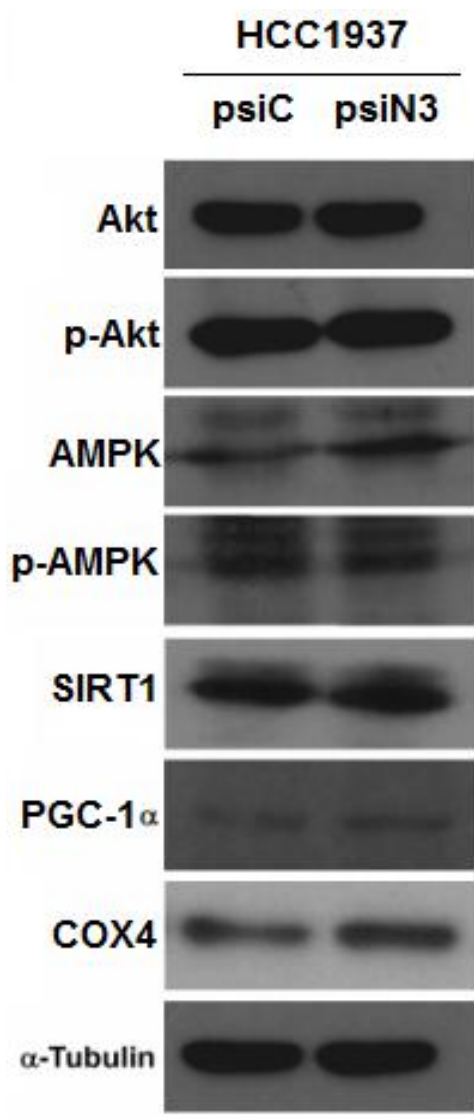

$\mathbf{e}$

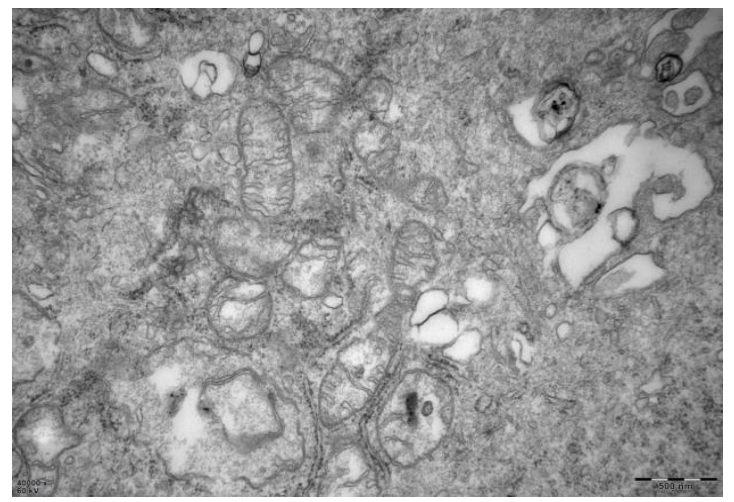

d
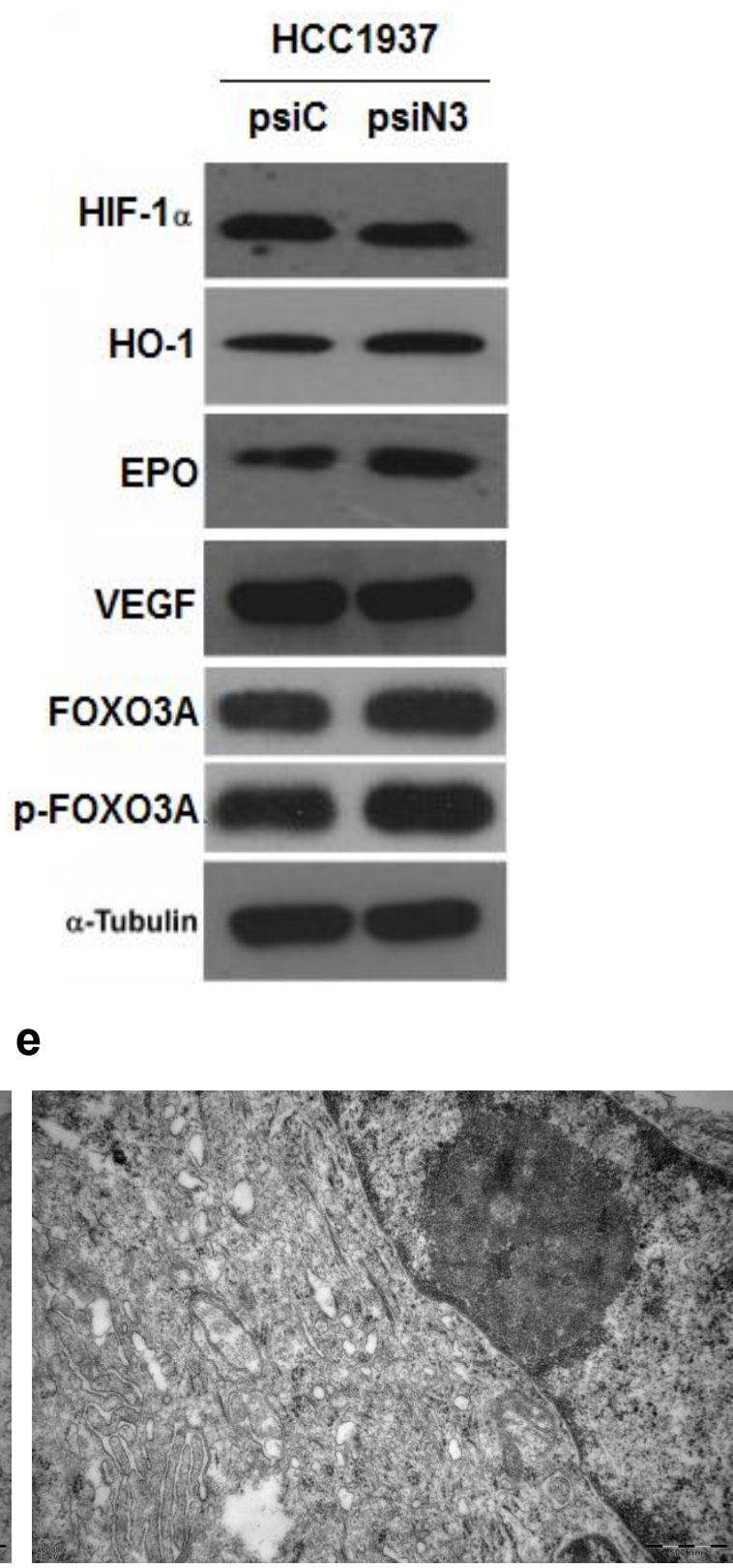

f

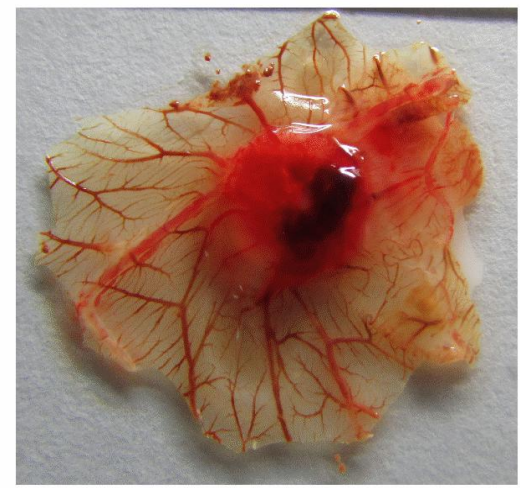


Fig. 4

Effects of NOS2 knockdown on the AMPK signalling pathway, mitochondrial biogenesis, and angiogenesis in HCC1937::psiC/psiN3 cells. a AKT, AMPK, SIRT1, PGC-1 $\alpha$, and COX4 mRNA levels; b HIF-1a, HO-1, EPO, VEGF, and FoxO3a mRNA; c AKT, p-AKT, AMPK, p-AMPK, SIRT1, PGC-1a, and COX4 levels; d HIF-1a, HO-1, EPO, VEGF, and FOXO3A levels. e The morphology of mitochondria (50,000X) in HCC1937::psiC (left) and in HCC1937:: psiN3 cells (right). f The chick chorioallantoic membrane assay in HCC1937::psiC (left) and in HCC1937::psiN3 cells (right).

\section{NOS2 knockdown suppresses tumour cell proliferation in vitro and retards tumour cell growth in nude mice}

To examine whether NOS knockdown would repress TNBC cell proliferation, we compared the growth rates between HCC1937:: psiN3 and HCC1937:: psiC cells. After culturing in vitro for $5 \mathrm{~d}$, a gradual decline in cell growth was observed for HCC1937::psiN3 cells. As shown in Fig. 5a, the growth rate of HCC1937::psiN3 cells was much lower than that of HCC1937::psiC cells, accounting for $35 \%$ of the decline in the absorbance at $490 \mathrm{~nm}$.

After culturing in vitro for 7-10 d, fewer colonies were counted on the plate of cultured HCC1937:: psiN3 cells than HCC1937:: psiC cells (Fig. 5b). The relative colony numbers were much lower for HCC1937:: psiN3 cells than HCC1937:: psiC cells (Fig. 5c). These results supported a correlation of repressed tumour proliferation with downregulated NOS 2 expression.

In the xenograft tumour-carrying nude mice, proliferation was observed for HCC1937::psiN3 cells, but not for HCC1937::psiC cells, suggesting that tumourigenicity was partially reversed upon NOS2 knockdown (Fig. 5d).

a

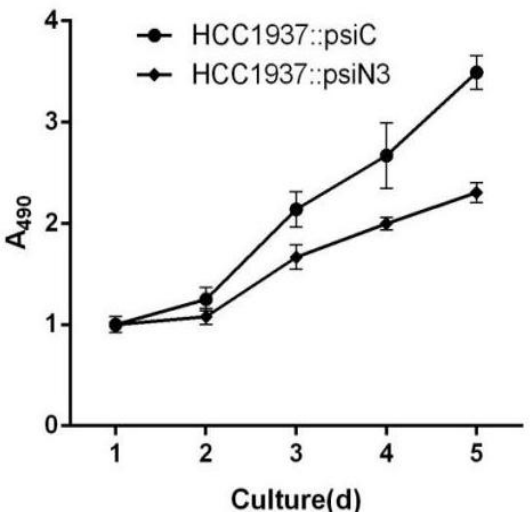

b HCC1937

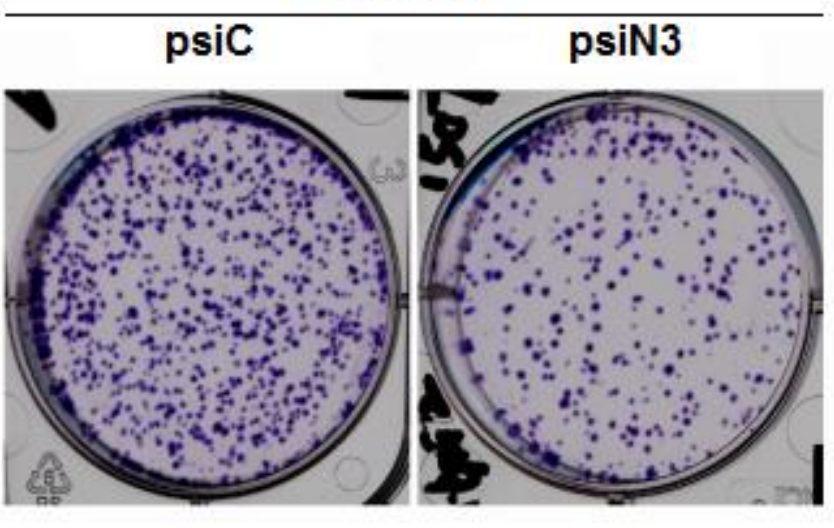


C

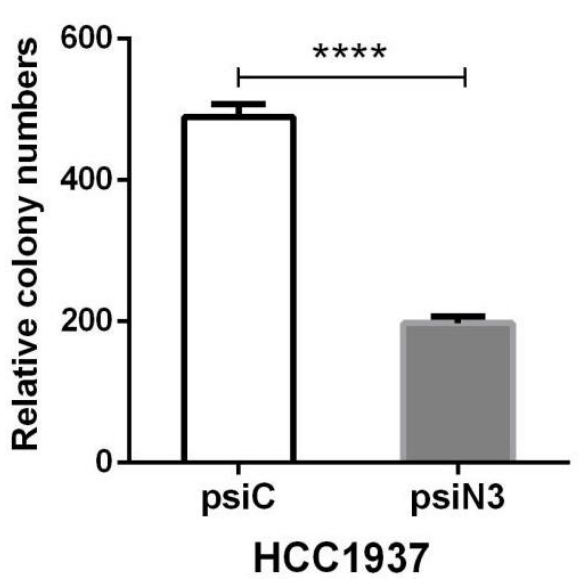

d

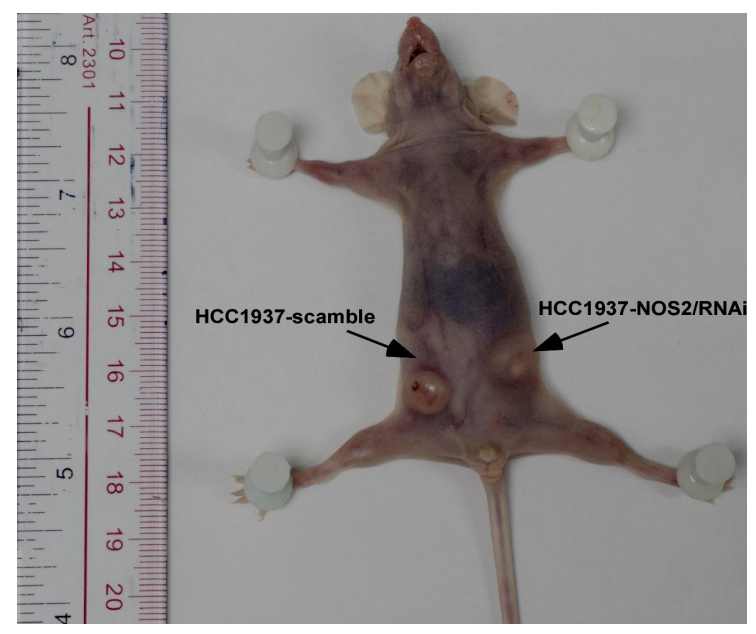

Fig. 5

Effects of NOS2 knockdown on cell proliferation in vitro and cell xenograft growth in a nude mouse. $\mathbf{a}$ the relative growth curve of HCC1937::psiC/psiN3 cells. $\mathbf{b}$ culture plates showing the colony numbers of HCC1937::psiC cells (left) and HCC1937::psiN3 cells (right). c the relative colony numbers of HCC1937::psiC/psiN3 cells. $\mathbf{d}$ the morphology of nude mice carrying the xenograft HCC1937::psiC/psiN3 cells.

\section{ESR1 knockdown decreases ER $\alpha$ and activates cell proliferative signalling with modulation of oncogenes and tumour suppressor genes}

To silence ESRl and mimic ER $\alpha$ inactivation, three recombinant ESR1-targeted siRNA expression constructs, psiRNA-ER1/2/3 (abbr. psiE1/2/3), were constructed by inserting one of three candidate shRNA fragments (the sequences were aligned in Methods), ESR 1 shRNA1/2/3, into the integration-type plasmid expression vector pSUPER-retro-puro.

After separately transferring those vehicles into MCF-10A cells, ESR $1 \mathrm{mRNA}$ and $\mathrm{ER} \alpha$ levels were quantified in MCF-10A::psiE1/2/3 cells transiently expressing ESR 1 shRNA. It was noted that ESR $1 \mathrm{mRNA}$ and ER $\alpha$ levels declined after transient infection of psiE1/2/3 into HCF-10A cells, as shown in Fig. 6a and 6b. From the expression levels, MCF-10A:: psiE3 cells showed the lowest ESR1 mRNA and lowest ER $\alpha$ levels. Thus, psiE3 was chosen for infection of MCF-10A cells to obtain stable ESR1 shRNA expression.

To create a milieu similar to oestrogenemia due to ER $\alpha$ dysfunction, MCF-10A::psiC/psiE3 cells were incubated with different E2 levels (10, 20, 40 , and $80 \mathrm{nM}$ ). After culturing for $24 \mathrm{~h}$, ROS levels were measured separately in the tested cells. As shown in Fig. 6c, the ROS burst occurred in an E2 concentration-dependent manner, among which $40 \mathrm{nM}$ E2 gave rise to the highest ROS levels. Thus, $40 \mathrm{nM}$ E2 was chosen for the subsequent incubation experiments.

To validate how can ESR 1 knockdown cause tumourigenic changes, the expression levels of some cell proliferative signalling transducer genes, 
oncogenes and tumour suppressor genes were quantified in MCF-10A::psiC/psiE3 cells. As results, ESR1 knockdown from MCF-10A cells could activate the cell proliferation-responsible $\mathrm{PI} 3 \mathrm{~K} / \mathrm{Akt} / \mathrm{mTOR}$ signalling pathway, in which Akt and p-Akt were upregulated but mTOR was unchanged. Interestingly, tumourigenic Ki67 and tumour suppressive E-cadherin were also synchronously upregulated, implying that tumourigenic and anti-tumourigenic roles coexisted in MCF-10A::psiE3 cells (Fig. 6d).

For other tumour markers and tumour suppressors, CD1 levels were higher in MCF-10A::psiE3 cells than in MCF-10A::psiE3 cells + E2, whereas the tumour suppressive CKI levels were lower in MCF-10A::psiE3 cells than in MCF-10A::psiE3 cells + E2 (Fig. 6e). Similarly, CD1 mRNA levels increased in MCF-10A::psiE3 cells, whereas CKI mRNA levels decreased in MCF-10A::psiE3 cells + E2 (Fig. 6f).

Though specific expression in TNBC, both RUNX1 and BCL11A mRNA levels were dramatically upregulated in MCF-10A::psiE3 cells, highlighting a typical feature of TNBC pathogenesis. Similarly, TP53BP1, which is underexpressed in most cases of TNBC, was also upregulated as a sign of DNA damage, suggesting an increase in DNA repair activity. In addition, ESRRG-encoding ERR $\gamma$ was upregulated with unknown functions (Fig. 6g).

These results clearly indicated that normal cells with ESR1 knockdown experienced a tumour-like change, such as an upregulation of oncogenes and a downregulation of tumour suppressor genes, in contrast to the pattern observed in tumour cells with NOS2 knockdown.

a

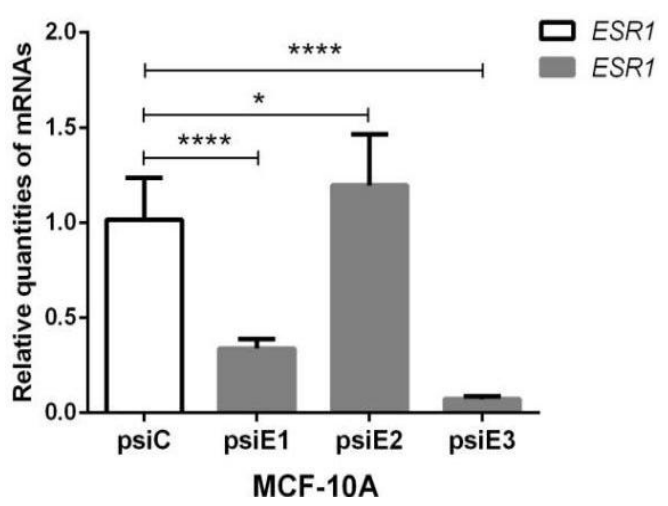

b

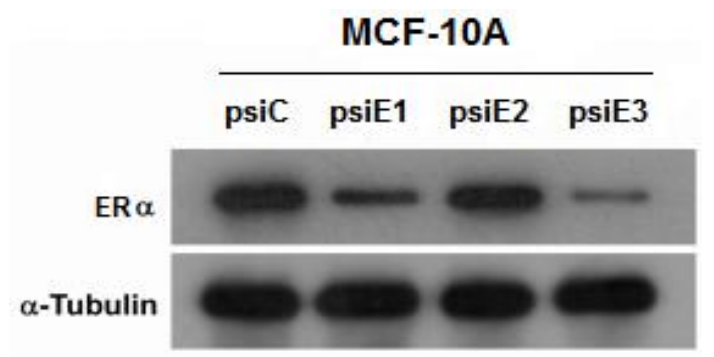




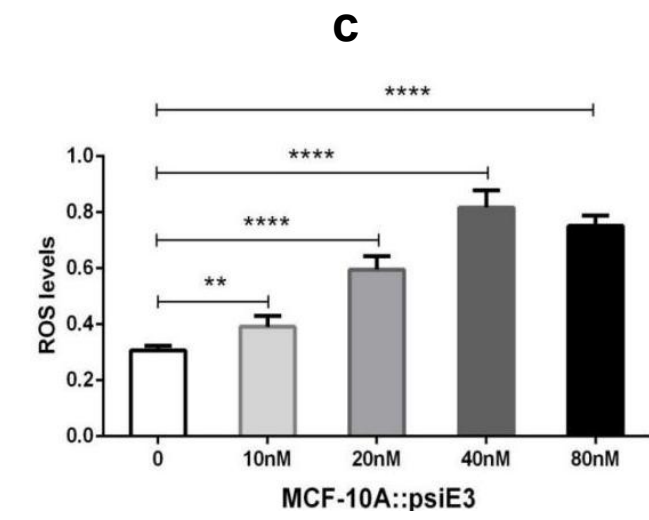

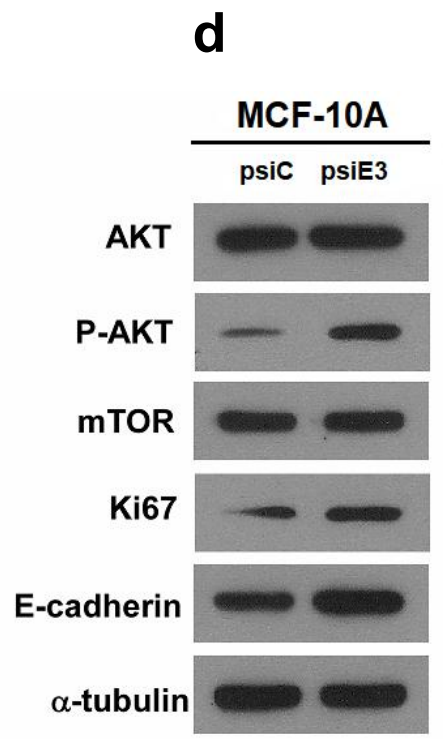

f e

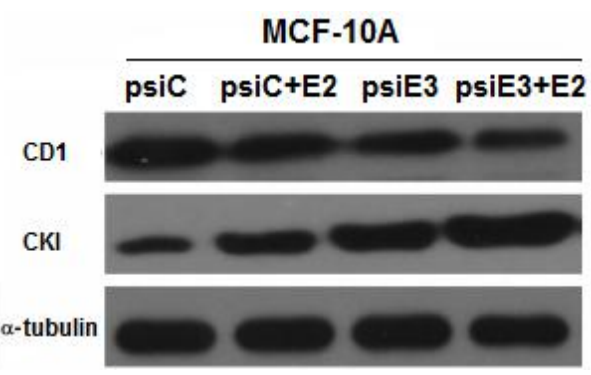

g
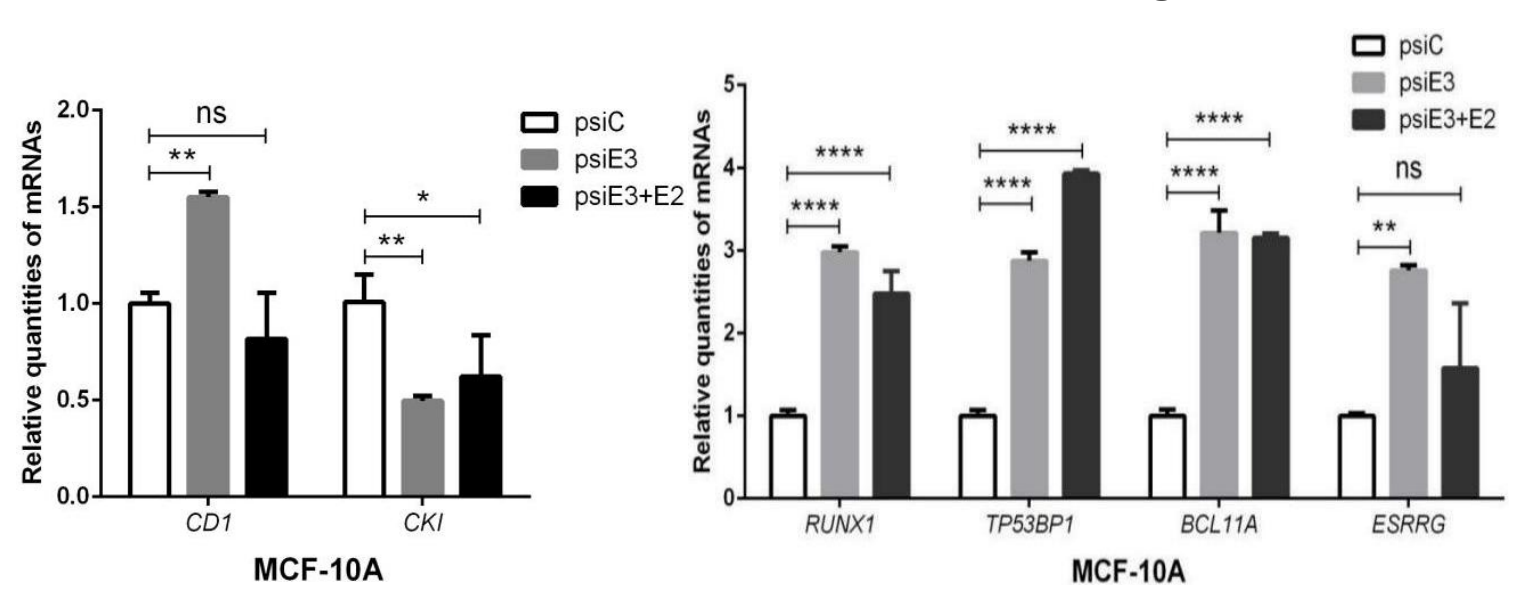

Fig. 6

The expression profiles of cell proliferative signalling transducer genes, oncogenes and tumour suppressor genes after shRNA-guided ESR1 knockdown in MCF-10A::psiC/psiE3 cells with or without E2. a ESR1 mRNA levels (transient expression); b ERa levels (transient expression); $\mathbf{c}$ ROS levels; $\mathbf{d}$ Akt, p-Akt, mTOR, Ki67 and E-cadherin levels; e CD1 and CKI levels; $\mathbf{f} C D 1$ and CKI mRNA levels; g RUNX1, TP53BP1, BCL11A and ESRRG mRNA levels.

\section{ESR1 knockdown enhances hypoxia, represses anti-oxidation and unalters mitochondrial biogenesis and metabolism}


To evaluate whether ESR2 knockdown would affect pro-inflammatory signaling, some pro-inflammatory cytokine and transcription factor mRNA levels were determined. Interestingly, ESR2 knockdown was shown to strengthen pro-inflammatory signalling in mammary epithelial cells, although this process did not involve typical immune cells. As illustrated in Fig. 7a, IL-6 mRNA and STAT3 mRNA levels were elevated in MCF-10A::psiE3 cells but declined in MCF-10A::psiE3 cells + E2, underscoring that ESR1 knockdown augmented pro-inflammatory signalling, whereas E2 treatment reversed the pro-inflammatory response and exerted an anti-inflammatory role.

Concomitantly, the upregulation of $H I F-1 \alpha$ reflecting hypoxia and $V E G F$ conferring angiogenesis, as well as the downregulation of $\mathrm{HO}-1$ and $E P O$ endowing anti-oxidative abilities, were observed in MCF-10A::psiE3 cells, implying an induction of $H I F-1 \alpha$ and $V E G F$ via pro-inflammation and a repression of $H O-1$ and $E P O$ by anti-inflammation. In contrast, a remarkable reversion of $H I F-1 \alpha$ and $V E G F$ overexpression was predominantly noticed in MCF-10A::psiE3 cells + E2 (Fig. 7b).

Accordingly, the mitochondrial biomarkers encoding $P G C-1 \alpha, C O X 1$ and COX4 mRNA were unchanged (Fig. 7c). GLUT1 mRNA responsible for intracellular glucose transport was upregulated in MCF-10A::psiE3 cells but downregulated in MCF-10A::psiE3+E2 cells. In contrast, MCT4 mRNA responsible for mitochondrial pyruvate transport was downregulated in MCF-10A::psiE3 cells but upregulated in MCF-10A::psiE3+E2 cells (Fig. 7d). These results indicated that ESR1 knockdown enhanced glycolysis in the cytosol but attenuated Krebs cycle reactions in mitochondria, although E2 could reverse those effects.

To investigate whether ER $\alpha$ downregulation would aggravate the inflammation-induced DNA damage, we evaluated the transcriptional profiles of 84 DNA damage pathway genes in MCF-10A::psiC/psiE3 cells by RT-PCR microarray. Consequently, DNA repairing-controlled ATR was upregulated 37.20-fold in MCF-10A::psiE3 cells compared with MCF-10A::psiC cells. Other genes involved in DNA damage and repair that were upregulated more than two-fold are illustrated in this clustering diagram in detail. The numbers of high-level mRNAs (red) were clearly higher in MCF-10A::psiE3 than in MCF-10A::psiC cells (Fig. 7e).

As verified by qPCR, an elevation of $A R T$ mRNA levels up to 10-folds was observed in MCF-10A::psiE3 cells compared with MCF-10A::psiC cells. Conversely, E2 treatment caused a significant decline in ART mRNA levels. Additionally, EPAS1 mRNA encoding HIF-2 $\alpha$ exhibited a similar pattern as $A R T$ mRNA, whereas ESR 2 mRNA encoding ER $\beta$ displayed a contrasting pattern to $A R T$ mRNA in MCF-10A::psiE3 cells, implying that the 
upregulation of HIF- $2 \alpha$ was changed similarly to HIF- $1 \alpha$, but ER $\beta$ without upregulation did not compensate for the deficiency of ER $\alpha$ after ESR1 knockdown.

a

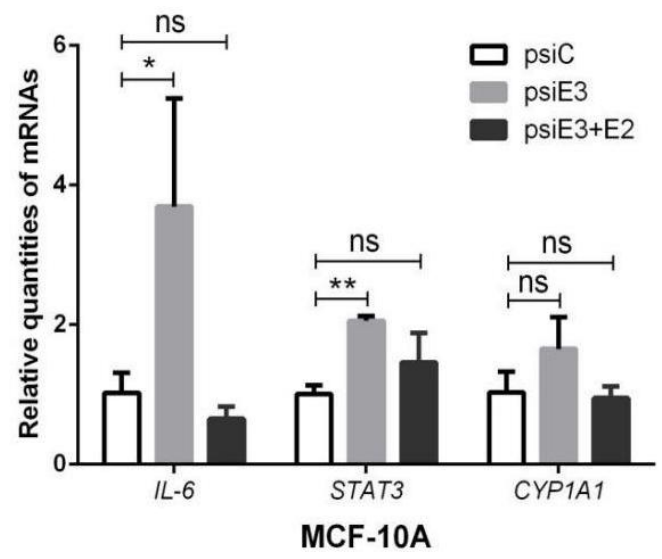

C b

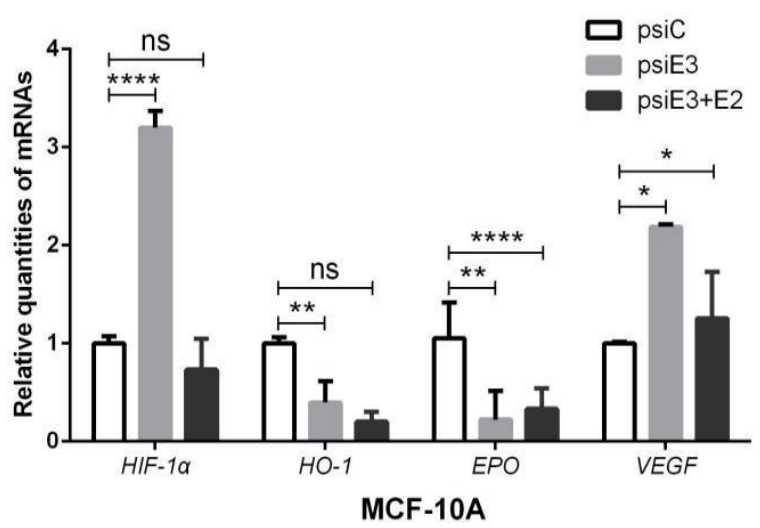

d
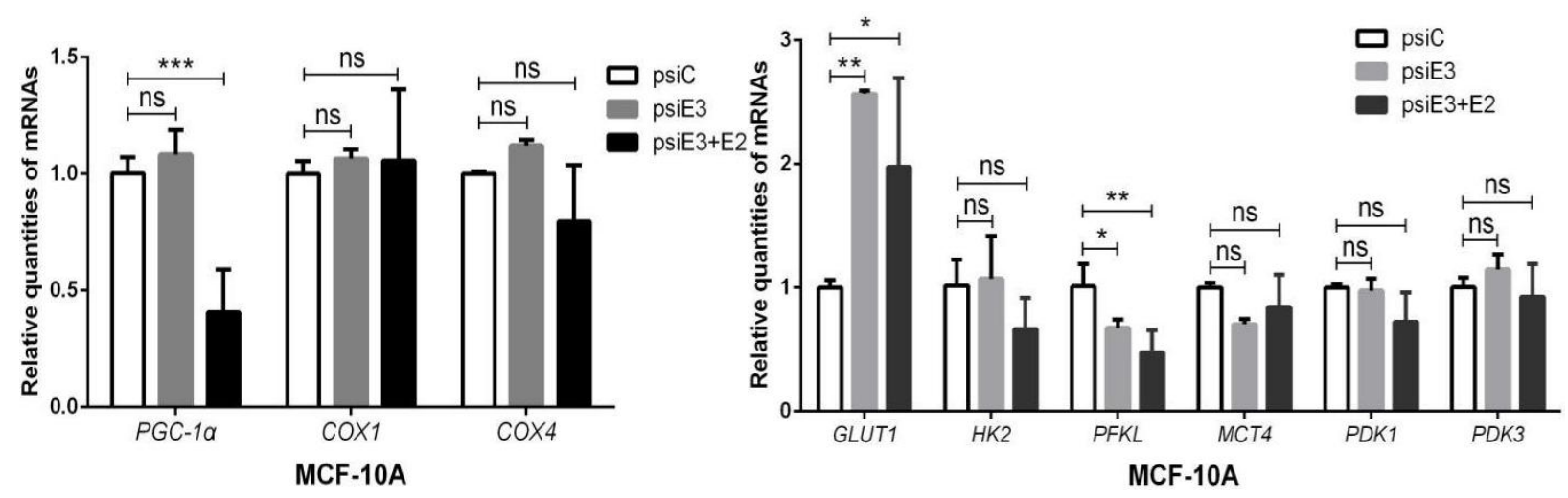

e

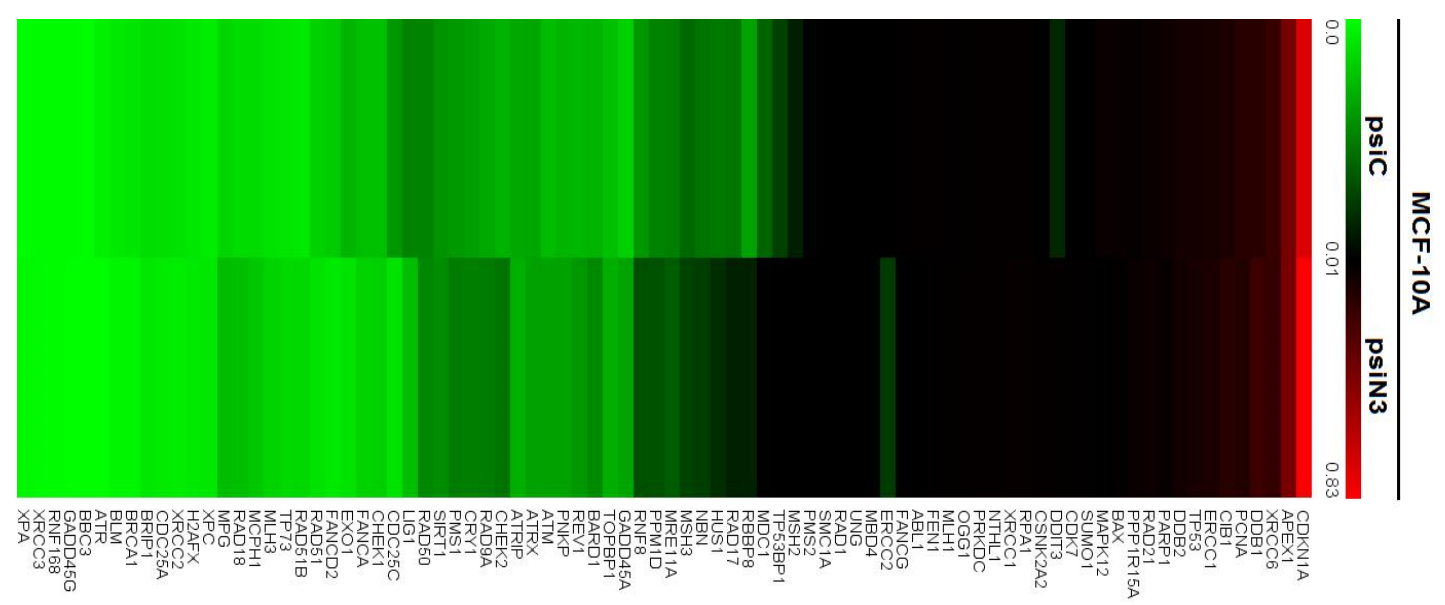




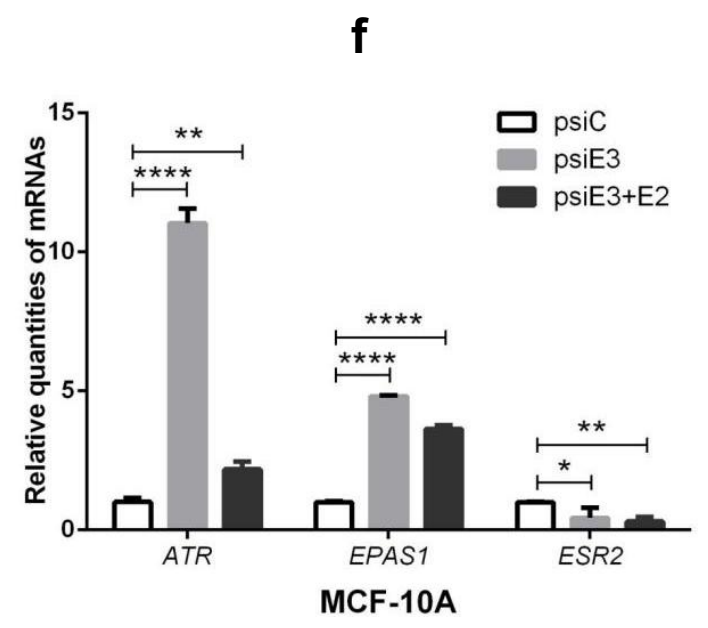

Fig. 7

The pro-inflammatory, hypoxic and oxidative responses in MCF-10A::psiC/psiE3 cells. a IL-6, STAT3, and CYP1A1 mRNA levels; b HIF-1a, HO-1, EPO, and VEGF mRNA levels; c PGC-1a, COX1, and COX4 mRNA levels; d GLUT1, HK2, MCT4, PDK1, and PDK3 mRNA levels; e DNA damage-involving mRNA levels (microarray). f ATR, EPAS1, ESR2 mRNA levels.

Cells with ESR1 knockdown neither displays proliferative features in vitro nor expresses tumourigenic phenotypes in vivo

According to our expectations, MCF-10A::psiE3 cells should grow faster than MCF-10A::psiC cells because the former exhibited tumourigenic features after ERS1 knockdown. However, ERS1 knockdown cells, either with or without addition of E2, showed fewer colonies (Fig. 8a), and MCF-10A::psiE3 cells with addition of E2 showed the least relative colony numbers among the compared groups (Fig. 8b). Regarding the MCF-10A::psiE3 cells, no tumour was seen on cell grafting nude mice, demonstrating that ESR 1 knockdown was not sufficient to confer an unlimited proliferative capacity to the grafted cells (Fig. 8c).

The dramatic decreases in the relative colony numbers following exposure of MCF-10A::psiC cells to E2 might be due to E2-triggered potent ROS burst and pronounced apoptosis induction. Correspondingly, E2-triggered ROS should induce SOD and CAT to scavenge ROS, which in turn downregulate SOD and CAT. Consistent with this reasoning, MCF-10A::psiC/psiE3 cells showed almost unchanged SOD and CAT levels, whereas MCF-10A::psiE3 cells + E2 exhibited much lower SOD and CAT levels (Fig. 8d and 8e).

Accordingly, the highest LA levels and the lowest ATP levels, so-called Warburg effects observed in the tumours, were measured in MCF-10A::psiE3 cells + E2 (Fig. 8f and 8g), which seemed to be an essential consequence of E2-driven oxidative stresses although ROS was eventually scavenged by antioxidant enzymes. 
a

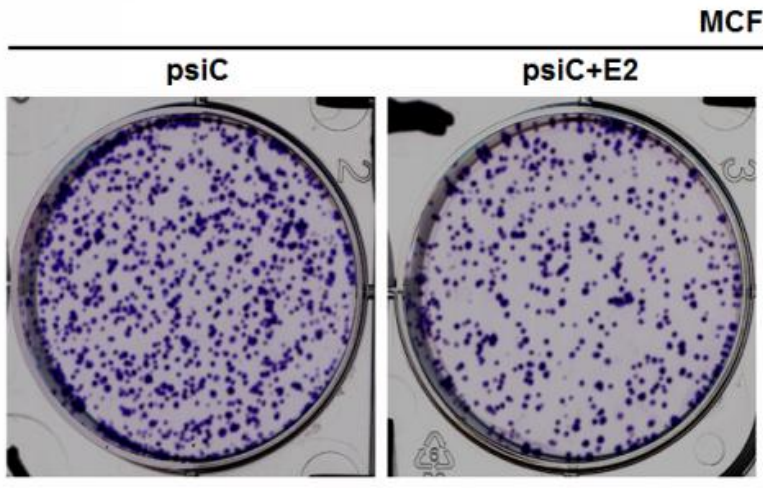

b

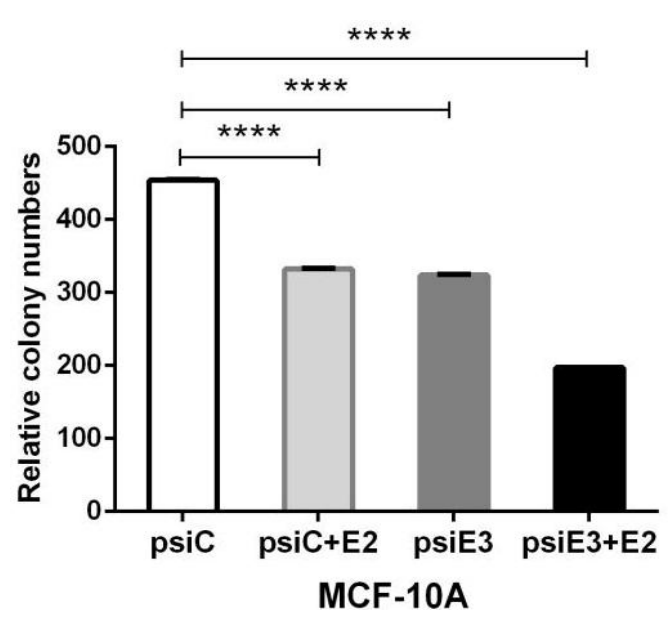

d
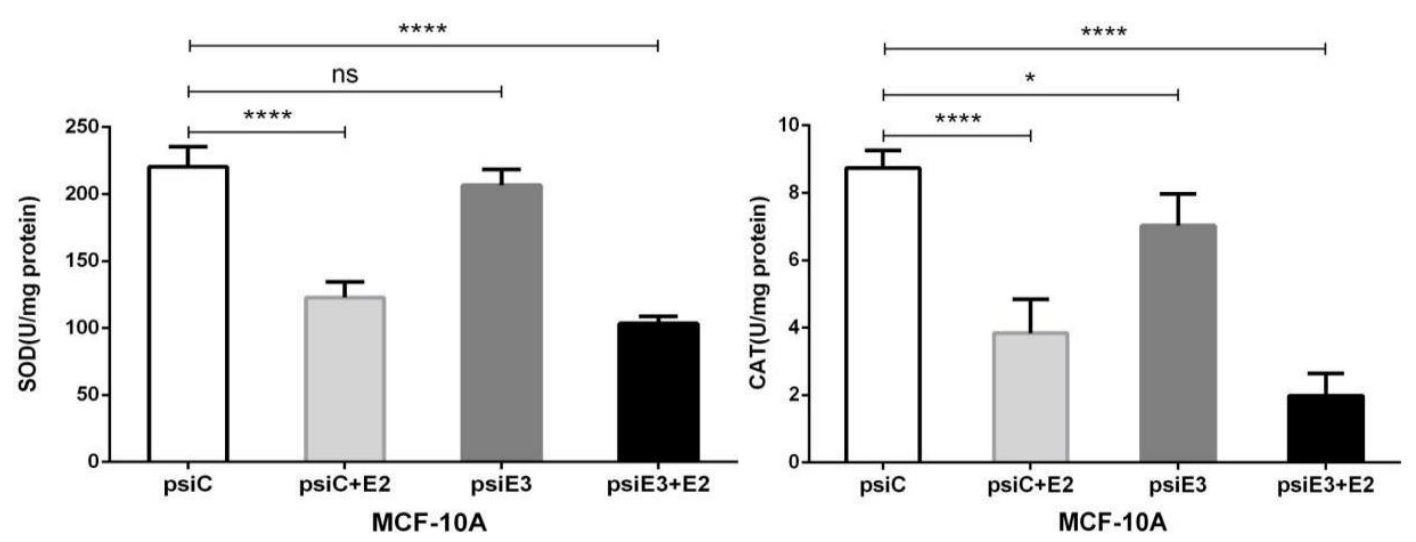
f

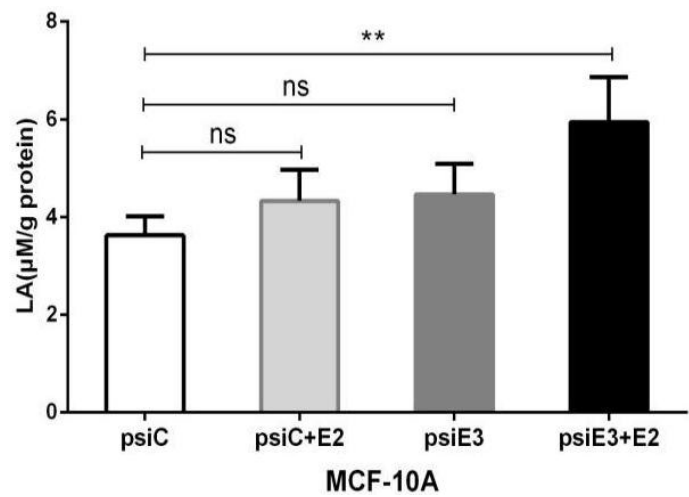

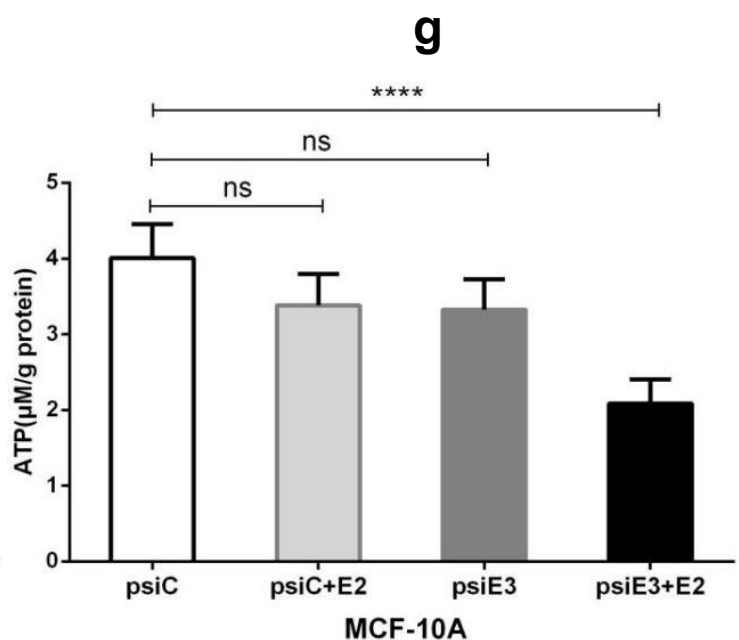

Fig. 8

Effects of ESR1 knockdown on cell proliferation in vitro and cell xenograft growth on the nude mouse. a culture plates showing colony numbers of MCF-10A::psiC, MCF-10A::psiC+E2, MCF-10A::psiE3, and MCF-10A::psiE3+E2 cells (from the left to the right). $\mathbf{b}$ the relative colony numbers of MCF-10A::psiC/psiE3 cells with or without E2. $\mathbf{c}$ the morphology of a nude mouse carrying xenograft MCF-10A::psiC/psiE3 cells, in which MCF-10A-scramble represents MCF-10A::psiC and MCF-10A-ERa/RNAi represents MCF-10A::psiE3; d SOD levels; e CAT levels; $\mathbf{f}$ LA levels; $g$ ATP levels.

These results demonstrated that ESR 1 knockdown only mimicked the tumourigenic features at the molecular level. Thus, tumourigenesis might require more time to evolve from molecular aspects to its phenotypic presentation.

\section{ESR1 knockdown reduces NO levels by dually downregulating iNOS and eNOS, coupled with compromised nitrosylation and apoptosis}

To figure out why tumour metabolic patterns were not transformed to tumour phenotypes as expectation, we assumed that the ESR1-modified cells might only exert the partial effects from ER $\alpha$ inactivation but not from iNOS activation. In other words, iNOS-derived NO could mediate many structural and functional changes of cellular components, which should be critical for tumourigenesis and carcinogenesis.

As shown in Fig. 9a, NO levels were reduced in MCF-10A:: psiE3 cells compared with MCF-10A::psiE3 cells + E2. Based on Fig. 9b and 9c, while unchanged or lower NOS $2 \mathrm{mRNA} / \mathrm{iNOS}$ and NOS3 mRNA/eNOS levels were measured in MCF-10A::psiE3 cells, higher NOS2 mRNA/iNOS and NOS3 mRNA/eNOS levels were quantified in MCF-10A::psiE3 cells + E2.

The lower NO levels should predispose lower NT levels because NT is a tyrosine residue modified by $\mathrm{ONOO}^{-}$derived from $\mathrm{NO}$ and ROS reactions. As observed, MCF-10A::psiC/psiE3 cells + E2 displayed lower NT levels (Fig. 9d). Similarly, MCF-10A::psiC/psiE3 + E2 cells exhibited a reduced extent of apoptotic induction (Fig. 9e). 


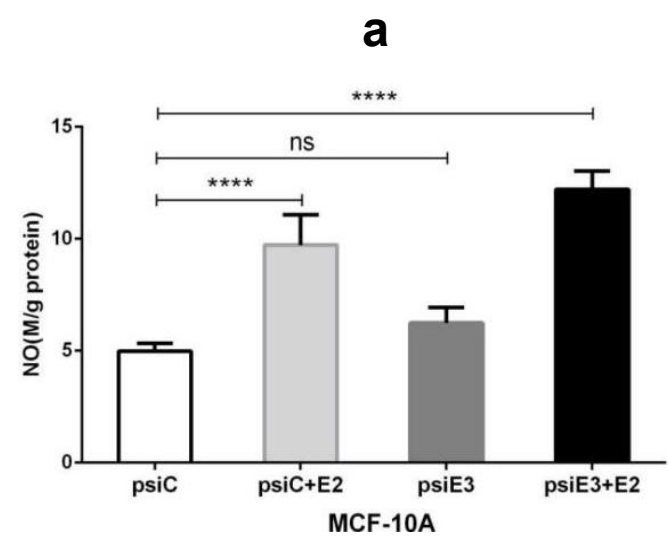

b

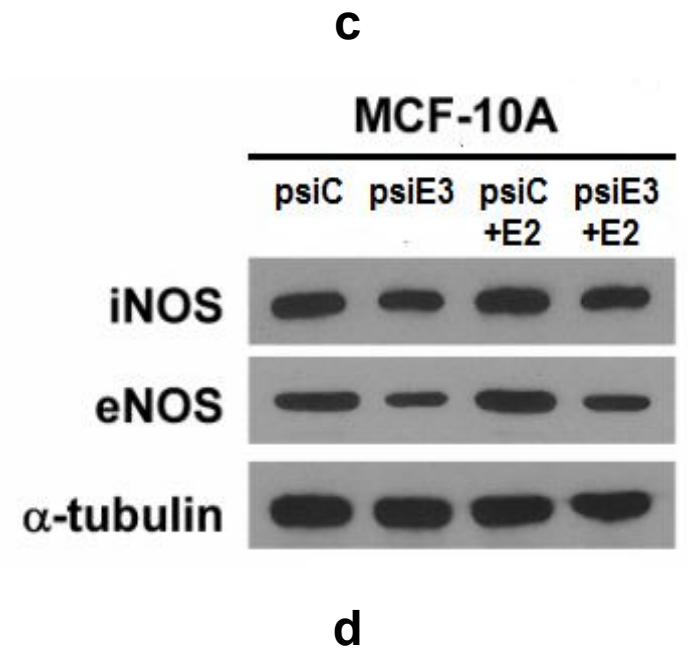

MCF-10A

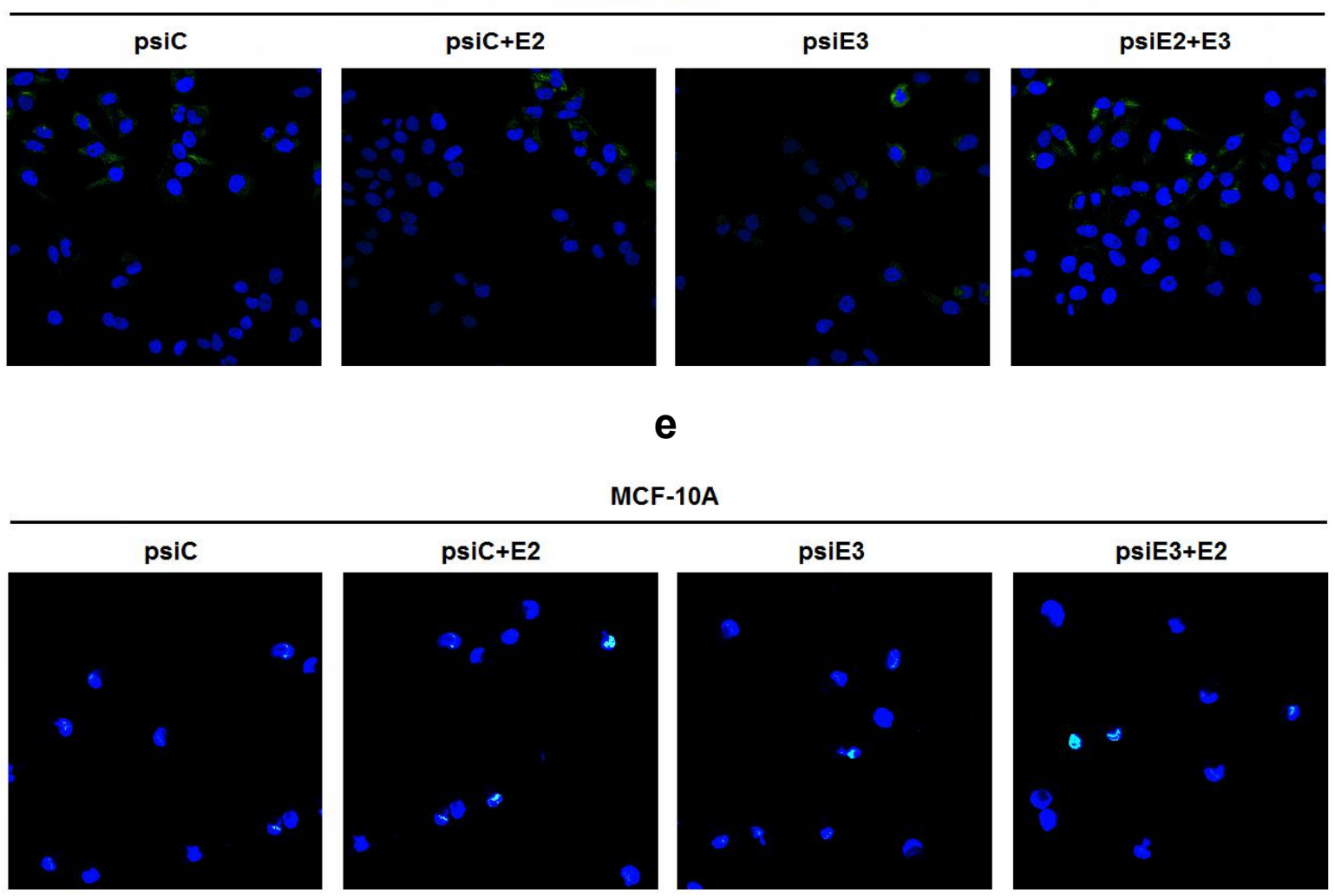

Fig. 9 
The effects of ESR1 knockdown with or without E2 on nitrosative stress, oxidative stress, and cell death in MCF-10A::psiC/psiE3 cells. a NO levels; b NOS2 and NOS3 mRNA levels; c iNOS and eNOS levels; $\mathbf{d}$ SOD levels; e CAT levels; $\mathbf{f}$ nitration in MCF-10A::psiC, MCF-10A::psiC+E2, MCF-10A::psiE3, MCF-10A:: psiE3+E3 cells (from the left panel to the right panel); $\mathbf{g}$ apoptosis in MCF-10A::psiC, MCF-10A::psiC+E2, MCF-10A::psiE3, MCF-10A::psiE3+E3 cells (from the left panel to the right panel).

These results indicated that no assistance with iNOS induction, ESR1 knockdown alone might be insufficient to allow the conversion of normal cell phenotypes into tumour cell phenotypes.

\section{Discussion}

To validate whether inflammation-driven NOS2/iNOS overexpression would be a point of origin for the initiation and maintenance of TNBC, we downregulated NOS2/iNOS in TNBC cells by shRNA-guided knockdown. NOS2/iNOS downregulation resulted in NOS3/eNOS upregulation, which compromised inflammatory responses, augmented mitochondrial biogenesis, and alleviated Warburg effects by converting the metabolism from anaerobic glycolysis to aerobic oxidative phosphorylation. These results were reverse-correlated with that of the transformation of normal cells to cancer cells accompanied by a switch from oxidative phosphorylation to glycolysis [25]. As expected for the mitigation of tumourigenesis, NOS 2 knockdown not only downregulated the special TNBC biomarkers $R U N X 1$ and $B C L 11 A$ but also the tumour marker CD1, and it upregulated the tumour suppressor CKI, indicating that NOS 2 knockdown partially reversed the tumourigenic features of TNBC cells.

Similar to NOS2 knockdown, the iNOS inhibitor $\mathrm{N}^{\mathrm{G}}$-monomethyl- $L$ arginine monoacetate (L-NMMA) blocked the tumour-like hyperplasia in our previous observations [26]. As further evidence, the pan-NOS inhibitor L-NMMA and the selective iNOS inhibitor $1400 \mathrm{~W}$ have been confirmed to diminish cell proliferation, cancer stem cell self-renewal, and cell migration in vitro [27]. These results strongly supported the conclusion that the switch of iNOS can serve as a critical check-point towards mammary differentiation or tumourigenesis. For other tumours, iNOS overexpression can result in human colon adenoma [28]; iNOS shows enhanced KRAS-induced lung carcinogenesis, inflammation and microRNA-21 expression [29]; and glioma stem cell proliferation and tumour growth are promoted by iNOS [30].

In contrast, the sustained induction of NOS2/iNOS leads to a potent NO burst, which enhances 3-nitration on tyrosine residues and S-nitrosylation on cysteine residues in proteins and enzymes, thereby inactivating them, including $E R \alpha$ and other receptors. Evidence supporting this phenomenon suggests that NO-mediated ER $\alpha$ nitrosylation regulates oestrogen-dependent gene transcription [31]; E2 and protein nitrosylation are synchronously augmented in the vascular endothelium [32]; and iNOS is expressed 
in human breast tumours, where its presence correlates with the tumour grade in patients [33].

To mimic nitrosylation-mediated ER $\alpha$ dysfunction, we downregulated $E S R 1 / E R \alpha$ in mammary epithelial cells by shRNA-directed knockdown. The results contrasted with NOS 2 knockdown-mediated partial TNBC reversal, as ESR 1 knockdown preliminarily re-enacted mammary tumourigenesis, in which tumour-like anaerobic metabolism and proliferative signalling were examined. While RUNX1 and BCL11A showed a downregulation, we also noticed an upregulation of CD1 and downregulation of CKI, together with the pronounced Warburg effects and enhanced ROS burst. These results showing a conversion from aerobic glucose catabolism to anaerobic glucose catabolism coincided with the classic metabolic features of tumour cells [25].

The high levels of oestrogens were found to upregulate ER expression, which initiates HIF-1 $\alpha$-mediated hyperplasia involving VEGF upregulation [34]. Under healthy conditions, oestrogens can reduce lipopolysaccharide (LPS)-triggered inflammation [35] by modulating the inflammatory p38 mitogen-activated protein kinase $(\mathrm{MAPK}) /$ nuclear factor $\kappa \mathrm{B}(\mathrm{NF}-\kappa \mathrm{B})$ cascade [36]. E2-mediated activation of serum-glucocorticoid regulated kinase 1 (SGK1) is known to suppress LPS-mediated apoptosis and promote anti-inflammatory TH2 responses in decidual stromal cells [37]. Several studies have also shown that oestrogens can alleviate oxidative stress [38). The beneficial effects of oestrogens are also manifested in the longer lifespan of women than men [39].

Regarding metabolic patterns, NOS2 knockdown in tumour cells maximised the Warburg effect, whereas ESR1 knockdown in normal cells minimised it, implying that breast cancer could be reversed or re-enacted by a single gene knockdown. Importantly, NOS2/iNOS knockdown could be simulated by iNOS inhibition through the use of $1400 \mathrm{~W}$ or L-NMMA, which should provide a cheap and convenient procedure for the treatment of clinically intractable TNBS patients. Considering the effectiveness of L-NMMA in decreasing tumour growth and enhancing the survival rate in TNBC, some authors have proposed to initiate a targeted therapeutic clinical trial of L-NMMA [27].

MCF-10A::psiE3 cells, either as in vitro cultures or in vivo xenografts, were not observed to proliferate as intrinsic tumour cells. This unexpected result requires further validation, both technical and mechanistical. Nevertheless, there are some hints for deciphering the ESRR overexpression-induced inhibition of cell proliferation [40]. Alternatively, a mechanistic link from the unchanged production of $\mathrm{NO}$ and ROS to the non-proliferative growth would be also established in MCF-10A cells only with ESR1 knockdown because iNOS could not be activated under a non-inflammatory niche. 
Furthermore, no mutations of two tumor suppressor genes, BRCA2 and TP53, were detected (unpublished data), reflecting an anti-tumor activity in MCF-10A::psiE3 cells.

Importantly, we observed the upregulation of proliferative PI3K/Akt/mTOR signalling transducers, including Akt, pAkt and mTOR, implied that tumourigenesis might have been initiated and would also be progress. There is a correlation of the specific proliferation of breast cancer with the overexpression of the cell cycle-regulated protein $\mathrm{Ki} 67$ [41], and thus our results reinforce that $\mathrm{Ki} 67$ overexpression is relevant to $\mathrm{MCF}-10 \mathrm{~A}$ tumourigenesis. In contrast, we examined the unexpected upregulation of E-cadherin in MCF-10A cells with ESRl knockdown, which is conventionally considered to be a good prognostic marker in cancer. However, questions regarding the prognostic value of E-cadherin and $\beta$-catenin in ductal carcinomas indicate a complicated role of these molecules in breast cancer progression [42]. Therefore, the upregulation of E-cadherin might represent an essential response against tumourigenesis.

\section{Conclusions}

Taken together, our experimental data support an association of the pro-inflammatory response with breast cancer occurrence via NOS2 activation and $\mathrm{ER} \alpha$ inactivation. While overexpression of TNBC biomarkers can be reversed in human TNBC cells by NOS 2 knockdown, they can also be re-enacted in human mammary epithelial cells by ESR 1 knockdown. These results should provide insight into the thorough exploration of the mysterious breast cancer origin and help to establish a targeted drug therapy regime for intractable TNBC.

\section{Acknowledgements}

The authors thank Ms Wei-Xia Zeng in LangRi, Guangzhou, China for her assistance in the construction of the vectors for gene knockdown.

\section{Funding}

This work was funded by the National Science Foundation of China (81774041 to QPZ and 81673861 to CQL), Guangzhou Science and Technology Plan Project (20187010007); Guangdong Provincial Science and Technology Plan Project (2015B020234003, 2014B050502013), State Administration of Traditional Chinese Medicine International Cooperation Special Project of Chinese Medicine (GZYYGJ2017009), Guangdong Provincial Chinese Medicine Bureau Project (2016 No. 53), and YangFan Innovative and Entrepreneurial Research Team Project (2014YT02S008) to JPS. 


\section{Availability of data and materials}

The datasets used and/or analysed during the current study are available from the corresponding author upon reasonable request.

\section{Ethics approval and consent to participate}

All experiments were approved by The Animal Care Welfare Committee, Guangzhou University of Chinese Medicine, Guangzhou, China (No. SPF2015009). The experimental protocol complies with the requirements of animal ethics issued in The Guide for the Care and Use of Laboratory Animals, National Institute of Health (NIH), USA. The number of animals used for each experiment was minimised as much as possible, and every effort was made to reduce the chance of pain or suffering.

\section{Competing interests}

The authors declare that they have no competing interests.

\section{References}

1. McGuire A, Brown JA, Malone C, McLaughlin R, Kerin MJ. Effects of age on the detection and management of breast cancer. Cancers 2015;7:908-929.

2. Anderson WF, Katki HA, Rosenberg PS. Incidence of breast cancer in the United States: current and future trends. J Natl Cancer Inst 2011;103:1397-1402.

3. Kohler BA, Sherman RL, Howlader N, Jemal A, Ryerson AB, Henry KA, et al. Annual report to the nation on the status of cancer, 1975-2011, featuring incidence of breast cancer subtypes by race/ethnicity, poverty, and state. J Natl Cancer Inst 2015;107:djv048.

4. Rosenberg PS, Barker KA, Anderson WF. Oestrogen receptor status and the future burden of invasive and in situ breast cancers in the United States. J Natl Cancer Inst 2015;107:djv159.

5. Wooster R, Weber BL. Breast and ovarian cancer. N Engl J Med 2003;348:2339-2347.

6. Savage KI, Matchett KB, Barros EM, Cooper KM, Irwin GW, Gorski JJ, et al. BRCA1 deficiency exacerbates oestrogen-induced DNA damage and genomic instability. Cancer Res 2014;74:2773-2784.

7. Yue W, Wang JP, Li Y, Fan P, Liu G, Zhang N, et al. Effects of oestrogen on breast cancer development: role of oestrogen receptor independent mechanisms. Int $\mathrm{J}$ cancer 2010;127:1748-1757.

8. Yang J, Tan Q, Fu Q, Zhou Y, Hu Y, Tang S, et al. Gastrointestinal microbiome and breast cancer: correlations, mechanisms and potential clinical implications. Breast Cancer. 2017; $24: 220-228$.

9. Kwa M, Plottel CS, Blaser MJ, Adams S. The intestinal microbiome and oestrogen receptor-positive female breast cancer. JNCI J Natl Cancer Inst 2016;108:djw029.

10. Flores R, Shi JX, Fuhrman BJ, Xu X, Veenstra TD, Gail MH, et al. Fecal microbial determinants of fecal and systemic oestrogens and oestrogen metabolites: a cross-sectional study. J TransI Med. 2012;10:253.

11. Yager JD, Davidson NE. Estrogen carcinogenesis in breast cancer. N Engl J Med. 2006;354:270-282. 
12. Fuhrman BJ, Feigelson HS, Flores R, Gail MH, Xu X, Ravel J, et al. Associations of the fecal microbiome with urinary oestrogens and oestrogen metabolites in postmenopausal women. J Clin Endocrinol Metab 2014;99:4632-4640.

13. Mailander PC, Meza JL, Higginbotham S, Chakravarti D. Induction of A.T to G.C mutations by erroneous repair of depurinated DNA following oestrogen treatment of the mammary gland of ACl rats. J Steroid Biochem Mol Biol 2006;101:204-215.

14. Gaikwad NW, Yang L, Muti P, Meza JL, Pruthi S, Ingle JN, et al. The molecular etiology of breast cancer: evidence from biomarkers of risk. Int J Cancer 2008;122:1949-1957.

15. Liehr JG, Fang WF, Sirbasku DA, Ari-Ulubelen A. Carcinogenicity of catechol oestrogens in Syrian hamsters. J. Steroid Biochem 1986;24:353-356.

16. Newbold RR, Liehr JG. Induction of uterine adenocarcinoma in CD-1 mice by catechol oestrogens. Cancer Res 2000;60:235-237.

17. Rogan EG, Badawi AF, Devanesan PD, Meza JL, Edney JA, West WW, et al. Relative imbalances in oestrogen metabolism and conjugation in breast tissue of women with carcinoma: potential biomarkers of susceptibility to cancer. Carcinogenesis 2003;24:697-702.

18. Huang J, Sun J, Chen Y, Song Y, Dong L, Zhan Q, et al. Analysis of multiplex endogenous oestrogen metabolites in human urine using ultra-fast liquid chromatography-tandem mass spectrometry: a case study for breast cancer. Anal Chim Acta 2012;711:60-68.

19. Bernard V, Kherra S, Francou B, Fagart J, Viengchareun S, Guéchot J, et al. Familial multiplicity of oestrogen insensitivity associated with a loss-of-function ESR1 mutation. J Clin Endocrinol Metab 2017;102:93-99.

20. Wang Q, Huang SQ, Li CQ, Xu Q, Zeng QP. Akkermansia muciniphila may determine chondroitin sulfate ameliorating or aggravating osteoarthritis. Front Microbiol 2017;8:1955.

21. Errico A. BCL11A-targeting triple-negative breast cancer? Nat Rev Clin Oncol. 2015;12:127.

22. Song H, Cicek MS, Dicks E, Harrington P, Ramus SJ, Cunningham JM, et al. The contribution of deleterious germline mutations in BRCA1, BRCA2 and the mismatch repair genes to ovarian cancer in the population. Human Mol Genet 2014;23:4703-4709.

23. Ferrari N, Mohammed ZM, Nixon C, Mason SM, Mallon E, McMillan DC, et al. Expression of RUNX1 correlates with poor patient prognosis in triple negative breast cancer. PLoS One 2014; 9:e100759.

24. Khaled WT, Choon Lee S, Stingl J, Chen X, Raza Ali H, Rueda OM, et al. BCL11A is a triple-negative breast cancer gene with critical functions in stem and progenitor cells. Nat Commun 2015; 6:5987.

25. Wu H, Ying M, Hu X. Lactic acidosis switches cancer cells from aerobic glycolysis back to dominant oxidative phosphorylation. Oncotarget 2016;7:40621-40629.

26. Wu P, Bao F, Xiao N, Wang DT, Zeng QP. Artemisinin and rapamycin compromise nitric oxide-driven and hypoxia-triggered acute articular synovitis in mice. Scientia Sinica Vitae 2012;42: 724-738.

27. Granados-Principal S, Liu Y, Guevara ML, Blanco E, Choi DS, Qian W, et al. Inhibition of iNOS as a novel effective targeted therapy against triple-negative breast cancer. Breast Cancer Res 2015;17:25.

28. Ambs S, Merriam WG, Bennett WP, Felley-Bosco E, Ogunfusika MO, Oser SM, et al. Frequent nitric oxide synthase-2 expression in human colon adenoma: implication for tumour angiogenesis and colon cancer progression. Cancer Res 1998;58:334-341.

29. Okayama H, Saito M, Oue N, Weiss JM, Stauffer J, Takenoshita S, et al. NOS2 enhances 
KRAS-induced lung carcinogenesis, inflammation and microRNA-21 expression. Int J Cancer 2013;132:9-18.

30. Eyler CE, Wu Q, Yan K, MacSwords JM, Chandler-Militello D, Misuraca KL, et al. Glioma stem cell proliferation and tumour growth are promoted by nitric oxide synthase-2. Cell. 2011;146:53-66.

31. Garbán HJ, Márquez-Garbán DC, Pietras RJ, Ignarro LJ. Rapid nitric oxide-mediated S-nitrosylation of oestrogen receptor: regulation of oestrogen-dependent gene transcription. Proc Natl Acad Sci USA 2005;102:2632-2636.

32. Chakrabarti S, Lekontseva O, Peters A, Davidge ST. 17beta-Estradiol induces protein S-nitrosylation in the endothelium. Cardiovasc Res 2010;85:796-805.

33. Thomsen LL, Miles DW, Happerfield L, Bobrow LG, Knowles RG, Moncada S. Nitric oxide synthase activity in human breast cancer. $\mathrm{Br} \mathrm{J}$ Cancer 1995;72:41-44.

34. Yang J, AITahan A, Jones DT, Buffa FM, Bridges E, Interiano RB, et al. Estrogen receptor- $\alpha$ directly regulates the hypoxia-inducible factor 1 pathway associated with antiestrogen response in breast cancer. Proc Natl Acad Sci USA 2015;112:15172-15177.

35. Blasco-Baque V, Serino M, Vergnes JN, Riant E, Loubieres P, Arnal JF, et al. High-fat diet induces periodontitis in mice through lipopolysaccharides (LPS) receptor signaling: protective action of oestrogens. PLoS One 2012;7:e48220.

36. Mu PW, Jiang P, Wang MM, Chen YM, Zheng SH, Tan Z, et al. Oestrogen exerts anti-inflammation via p38 MAPK/NF-KB cascade in adipocytes. Obes Res Clin Pract 2016;10:633-641.

37. Lou Y, Hu M, Wang Q, Yuan M, Wang N, Le F, et al. Estradiol suppresses TLR4-triggered apoptosis of decidual stromal cells and drives an anti-inflammatory TH2 shift by activating SGK1. Int J Biol Sci 2017;13:434-448.

38. Brinton LA, Lacey JV Jr, Trimble EL. Hormones and endometrial cancer--new data from the Million Women Study. Lancet 2005;365:1517-1518.

39. Borras C, Gambini J, Vina J. Mitochondrial oxidant generation is involved in determining why females live longer than males. Front Biosci 2007;12:1008-1013.

40. Cheung CP, Yu S, Wong KB, Chan LW, Lai FM, Wang X, et al. Expression and functional study of estrogen receptor-related receptors in human prostatic cells and tissues. J Clin Endocrinol Metab 2005;90:1830-1844.

41. Pathmanathan N, Balleine RL. Ki67 and proliferation in breast cancer. J Clin Pathol. 2013;66(6):512-516.

42. Borcherding N, Cole K, Kluz P, Jorgensen M, Kolb R, Bellizzi A, et al. Reevaluating E-cadherin and $\beta$-catenin: a pan-cancer proteomic approach with an emphasis on breast cancer. Am J Pathol. 2018; pii: S0002-9440(18)30113-5. 